\title{
High-Fidelity Computational Aerodynamics of Multi-Rotor Unmanned Aerial Vehicles
}

\author{
Patricia Ventura Diaz* and Seokkwan Yoon ${ }^{\dagger}$ \\ NASA Ames Research Center, Moffett Field, California, 94035, USA
}

\begin{abstract}
High-fidelity Computational Fluid Dynamics (CFD) simulations have been carried out for several multi-rotor Unmanned Aerial Vehicles (UAVs). Three vehicles have been studied: the classic quadcopter DJI Phantom 3, an unconventional quadcopter specialized for forward flight, the SUI Endurance, and an innovative concept for Urban Air Mobility (UAM), the Elytron 4S UAV. The three-dimensional unsteady Navier-Stokes equations are solved on overset grids using high-order accurate schemes, dual-time stepping, and a hybrid turbulence model. The DJI Phantom 3 is simulated with different rotors and with both a simplified airframe and the real airframe including landing gear and a camera. The effects of weather are studied for the DJI Phantom 3 quadcopter in hover. The SUI Endurance original design is compared in forward flight to a new configuration conceived by the authors, the hybrid configuration, which gives a large improvement in forward thrust. The Elytron 4S UAV is simulated in helicopter mode and in airplane mode. Understanding the complex flows in multi-rotor vehicles will help design quieter, safer, and more efficient future drones and UAM vehicles.
\end{abstract}

\section{Introduction}

Unmanned Aerial Vehicles (UAVs) have grown very popular over the last decade. While originally UAVs were designed for military applications, their use has rapidly expanded to the civil market. The unique ability of vertical lift vehicles to hover has great potential for human and cargo transportation, delivery systems, inspection and surveillance missions, and disaster relief. Since unmanned vehicles are sized and optimized for particular missions, modern low-fidelity conceptual design and sizing tools that have been used for the design of large helicopters can also be used for the design of multi-rotor craft. However, there are aerodynamic features of these multi-rotor vehicles that can be difficult to account for with these low-fidelity tools, unless there is a method to calibrate the tools. Compared to single rotor systems, multi-rotor vehicles offer an advantage in lifting capacity ${ }^{1}$ because the size of a single rotor is limited by the tip speed and structural mechanics.

Accurate prediction of rotorcraft performance continues to be challenging. The flows are inherently unsteady, nonlinear and complex. For instance, a rotor blade can encounter its own tip vortex and the tip vortices of other blades. It is even more difficult when there are aerodynamic interactions between multiple rotors and fuselage because of the close proximity of all of these components. High-fidelity Computational Fluid Dynamics (CFD) methods may offer an advantage over low-fidelity tools when investigations of interactional aerodynamics of multi-rotor vehicles are required. High-fidelity CFD can also provide information to calibrate low-fidelity design tools to account for aerodynamic interactions.

The objective of the present work is to demonstrate a high-fidelity computational simulation capability to study the aerodynamics of multi-rotor unmanned aerial vehicles. Two common quadcopters - the DJI Phantom 3 and the Straight Up Imaging (SUI) Endurance - and an innovative UAV concept - the Elytron $4 \mathrm{~S}$ UAV - are shown in figure 1. In this study, the DJI Phantom 3, the SUI Endurance, and the Elytron $4 \mathrm{~S}$ UAV are simulated using a high-order accurate CFD solver. The complex flows for the vehicles in different configurations are analyzed and compared.

\footnotetext{
*Science \& Technology Corporation.

†NASA Advanced Supercomputing Division.
} 


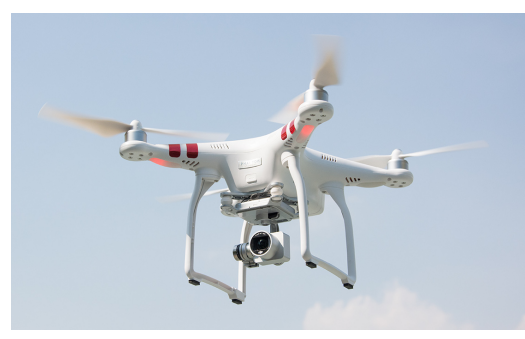

(a) DJI Phantom 3.

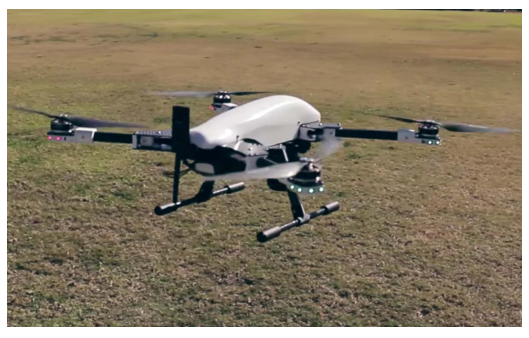

(b) SUI Endurance.

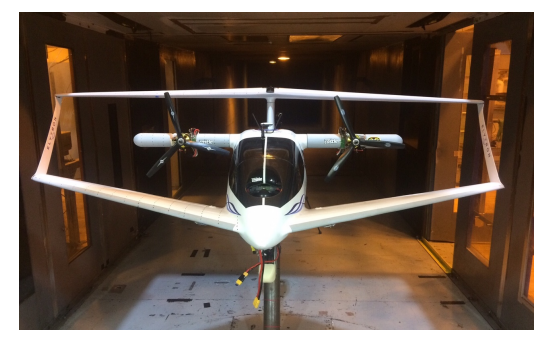

(c) Elytron 4S UAV.

Figure 1. DJI Phantom 3 quadcopter, SUI Endurance quadcopter and Elytron 4S UAV.

The DJI Phantom 3 is an example of a classic commercial quadcopter design with a symmetric X-shaped airframe. It is a good configuration for hover and control above static or slow moving objects, and it can be used to record high quality videos. Previous studies ${ }^{1,2}$ simulated the flow in hover for the simplified DJI Phantom 3 configuration ${ }^{\text {a }}$. Here, the complete configuration is simulated in hover and compared to the simplified configuration. The simplified configuration has been simulated with two different rotors: Floureon's carbon fiber $(\mathrm{CF})$ replica of the 9443 rotor blades, and the original DJI Phantom 3 rotor blades. From now on, the configuration with the original Phantom 3 blades and complete airframe - body, battery, landing gear and camera - will be referred to as Phantom 3 complete configuration. The system with the simplified airframe - only body - and the original DJI Phantom 3 rotor blades will be called Phantom 3 simplified configuration. Finally, the configuration with the simplified airframe and the carbon fiber $(\mathrm{CF})$ replica blades will be referred to as Phantom 3 simplified CF configuration. The effects of sudden wind gusts during hover have also been simulated for the complete configuration. To the authors' knowledge, this is the first time UAVs have been simulated under weather conditions.

The SUI Endurance ${ }^{\mathrm{b}}$ is an example of a forward flight quadcopter design with an elongated airplanelike airframe that can be used in missions where the UAV will be operating most of its flight envelope in forward flight mode. Thus, it is designed for faster speeds in forward flight than a regular quadcopter, and it can be used for cargo transportation or video recording of fast-moving objects. This study focuses on the performances and characteristics of the flow in forward flight for the SUI Endurance. Again, two configurations have been generated. The first configuration, SUI standard, is the original configuration for SUI Endurance UAV, where the four propellers are over-mounted. In the second configuration, SUI hybrid, the fore rotors are under-mounted and the aft rotors are over-mounted. The performances of the two configurations are compared in forward flight.

The Elytron 4S UAV - or Elytron for short - is the UAV scaled model of the future Urban Air Mobility (UAM), popularly known as "flying car", Elytron $4 \mathrm{~S}^{\mathrm{c}}$. The innovative design of the Elytron includes a tiltwing for Vertical Take-Off and Landing (VTOL) and a box wing for reducing induced drag. The Elytron $4 \mathrm{~S}$ UAV is simulated in order to analyze the flow structures and the stability of this configuration and to study the feasibility of the UAM Elytron $4 \mathrm{~S}$.

\section{Numerical Approach}

The flow solver used in this study is NASA's OVERFLOW ${ }^{3,4}$ high-fidelity CFD solver. OVERFLOW is a finite-difference, overset grid, high-order accurate Navier-Stokes flow solver. NASA's Chimera Grid Tools $(\mathrm{CGT})^{5}$ overset grid generation software is used for generating the overset grids of the complete vehicles. Body-fitted curvilinear near-body (NB) grids are generated using CGT. The computational domain is completed with the generation of Cartesian off-body (OB) grids; these are automatically generated prior to grid assembly using the Domain Connectivity Framework (DCF) in OVERFLOW-D mode. The current time-accurate approach consists of an inertial coordinate system in which near-body curvilinear O-grids for the rotor blades rotate through the fixed off-body Cartesian grid system.

\footnotetext{
ahttps://www nasa.gov/image-feature/ames/exploring-drone-aerodynamics-with-computers

bhttp://www.straightupimaging.com/

${ }^{\mathrm{c}}$ http://www. converticopter.com/
} 


\section{A. Overset Grid Generation}

The overset grid generation using CGT can be divided into the following steps: geometry processing, surface grid generation, volume grid generation, and domain connectivity. ${ }^{6}$ The geometry is usually obtained from a Computer Aided Design (CAD) model or a 3D-scanning point cloud. Once the geometry is processed as a triangulation reference surface, overlapping hyperbolic or algebraic surface grids are generated. The generation of surface grids is the step that requires the most manual effort and experience from the user.

With sufficient overlap between surface grids, the volume grids can be created easily with hyperbolic marching methods out to a fixed distance from the surface. Such methods provide orthogonal grids with tight clustering characteristics at the wall, which is essential for accurately capturing the boundary layer in viscous flow computations. The distance is chosen such that the outer boundaries of the near-body volume grids are well clear off the boundary layer. The near-body grids are then embedded inside off-body Cartesian grids that extend to the far field.

Surface grid resolution on the rotor blades is clustered in the chordwise direction near the airfoils leading and trailing edges to accurately resolve large pressure gradients. The spanwise resolution is clustered near the root and the tip. There is not an established practice for generating the grids for the airframes, as each case has its own topology. In general, clustering near corners and high curvature regions is good practice. The normal grid spacing of all surfaces maintains a $y^{+}<1$.

Off-body Cartesian grids with uniform spacing surround the near-body grids to resolve the wake region of interest. Coarser Cartesian grids efficiently expand the grid system to the far field, where each successive Cartesian grid is twice as coarse as its previous neighbor. The far-field boundary is 25 rotor radii away from the center of the vehicle in all directions. The resolved wake region has a uniform grid spacing of $10 \%$ of the tip chord length $c_{t i p}$.

By using a trimmed approach, the domain connectivity step is robust and highly automated: no hole cutting is required other than that on the off-body Cartesian grids. In this study, the X-ray hole cutting method is used. An X-ray object is created for every component in the geometry (i.e. the blades, the airframe, the landing gear, etc.). The user has to supply the list of meshes that each X-ray object is allowed to cut, and an offset distance with which to grow each hole away from the body. The hole cutting process is performed at each time step within the flow solver, allowing for the rotation of the blades.

\section{DJI Phantom 3 overset grids}

The first vehicle for analysis in this study is the complete DJI Phantom 3. The quadcopter system is constructed by incorporating the rotors to the X-shaped airframe in diagonal-opposed clock-wise (CW) and counter-clock-wise (CCW) positions for torque cancellation.

As mentioned in the introduction, three configurations are studied here, the complete Phantom 3 , the simplified Phantom 3, and the simplified CF Phantom 3; see figures 2 and 3.

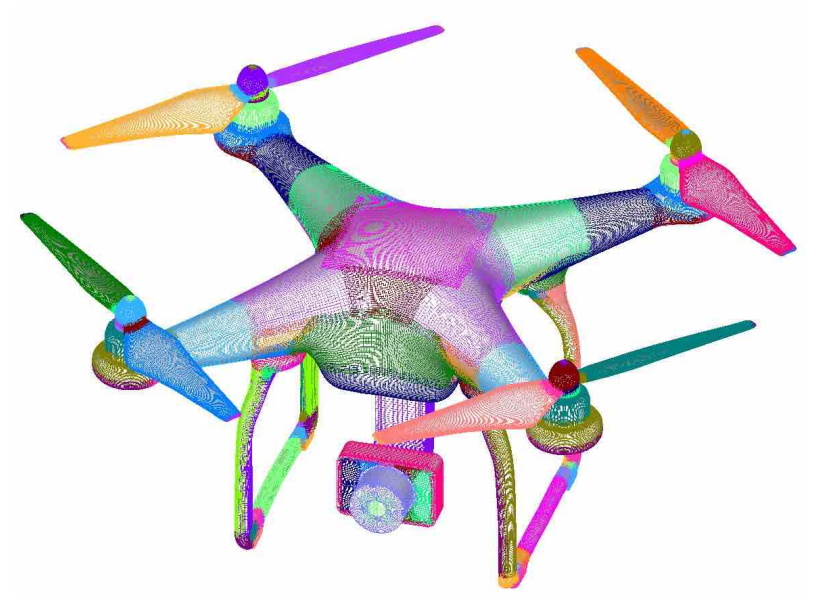

(a) Complete DJI Phantom 3 overset surface grids.

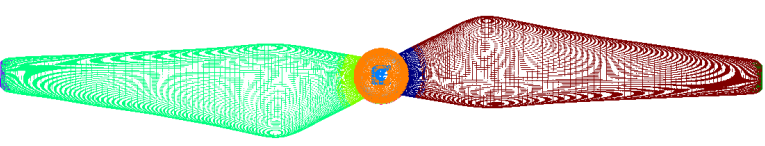

(b) DJI Phantom 3 plastic rotor blades overset surface grids.

Figure 2. Complete DJI Phantom 3 quadcopter overset surface grids. 
The complete Phantom 3 consists of the original Phantom 3 airframe - an X-shaped airframe with landing gear, battery and camera - and the original Phantom 3 injection-molded flexible rotor blades. The complete airframe and the original propeller shapes have been obtained by NASA Ames Research Center using high resolution laser scan techniques. The simplified Phantom 3 configuration is composed of the original Phantom 3 plastic rotor blades and the simplified airframe - an X-shaped airframe without any other components. The simplified airframe has been modeled using high-order polynomials and CAD software. Finally, the simplified Phantom 3 CF configuration consists of the simplified airframe and Floureon's carbon fiber (CF) replica of the 9443 rotor blades that are compatible with the DJI Phantom 2 and Phantom 3. The rotor blade data was extracted from a high-resolution scan of the blade surfaces. NASA Langley tested the CF replica rotor blades in the Structural Acoustic Loads and Transmission anechoic chamber facility. ${ }^{7}$ Tests of Phantom 3 rotors at NASA Ames revealed that the original blades yield higher performance and efficiency than the rigid $\mathrm{CF}$ rotor blades. ${ }^{8}$

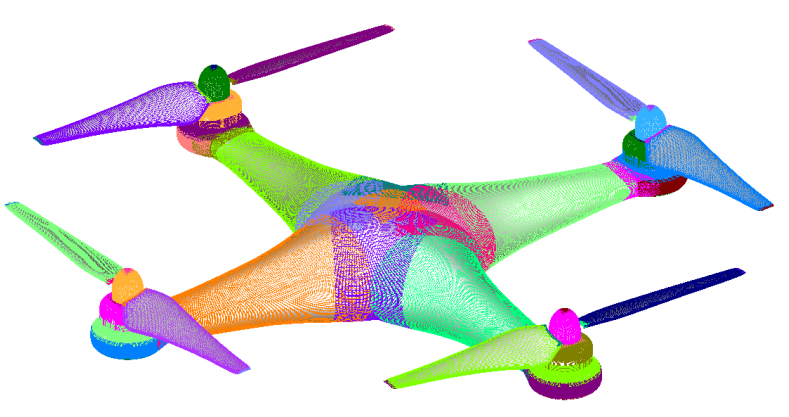

(a) Simplified CF Phantom 3 overset surface grids.

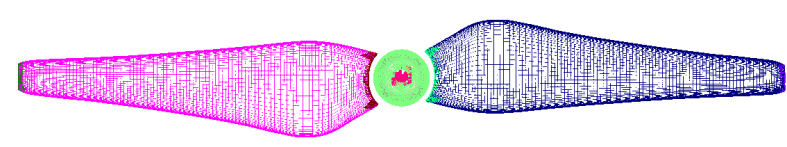

(b) Phantom CF rotor blades overset surface grids.

Figure 3. Simplified Phantom 3 quadcopter overset surface grids.

For the complete Phantom 3 airframe, the point cloud has been transformed into a CAD model and then discretized into a triangulation using commercial software. At this point, the triangulation can be imported into CGT and the overset grids generated as outlined previously.

In the case of the $\mathrm{CF}$ and the plastic propellers, airfoil profiles at different radii $r$ were calculated from the point cloud. The profiles were connected and smoothed, obtaining the whole blade. The blades are then attached at the center to a hub, conforming the rotor. Therefore, each rotor grid system consists of two blades attached to a hub. O-grids are used for the blades. Cap grids are generated for the blade tips and the hub axis. In the junction blade-hub, a collar grid is employed. The rotor blade has a radius of $R_{t i p}=0.12 \mathrm{~m}$ and a tip chord of $c_{t i p}=0.01 \mathrm{~m}$ approximately. The distance from the center of the vehicle to the rotational axis of each rotor is $L=0.175 \mathrm{~m}$. Figure 4 shows a comparison of blade characteristics between $\mathrm{CF}$ and factory blades. The chord length is normalized by the rotor radius. The CF rotor blade geometry has been modified in order to have higher performance. There exists a gap between the hub and blade, and the pitch angle has been increased to $4^{\circ}$ for better performance.

The resulting near-body grids for the complete Phantom 3 quadcopter consist of 202 overset grids. There are a total of 29 million grid points for the near-body grid system only. The grid system of off-body and near-body grids consists of 396 million grid points.

The near-body grids for the simplified Phantom 3 quadcopter consist of 86 overset grids, with 43 million grid points. The grid system of off-body and near-body grids has a total of 393 million grid points.

Finally, the simplified CF Phantom 3 quadcopter has 74 overset near-body grids with 40 million grid points. The complete grid system of near-body and off-body contains 387 million grid points.

\section{SUI Endurance overset grids}

The SUI Endurance quadcopter is the second vehicle studied. The model consists of four rotors, the airframe, the landing gear, the canards, and the camera support. The rotors are added to the airframe in diagonalopposed CW and CCW positions for torque cancellation. 


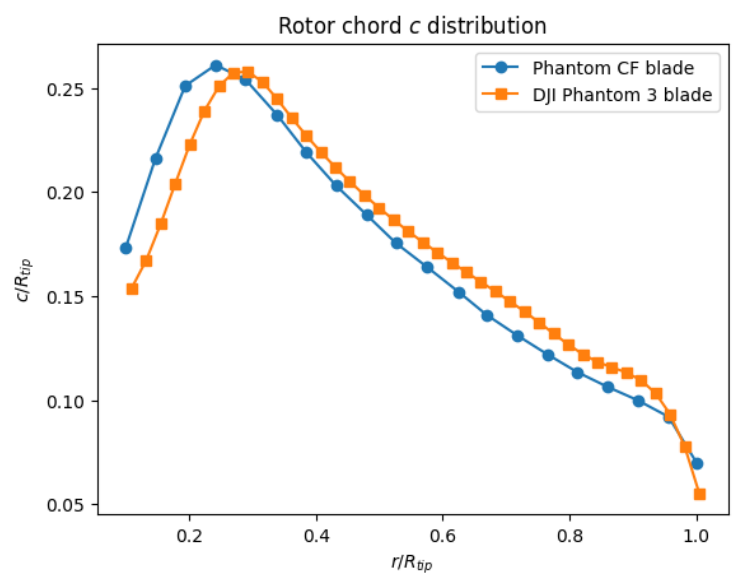

(a) Rotor chord distribution.

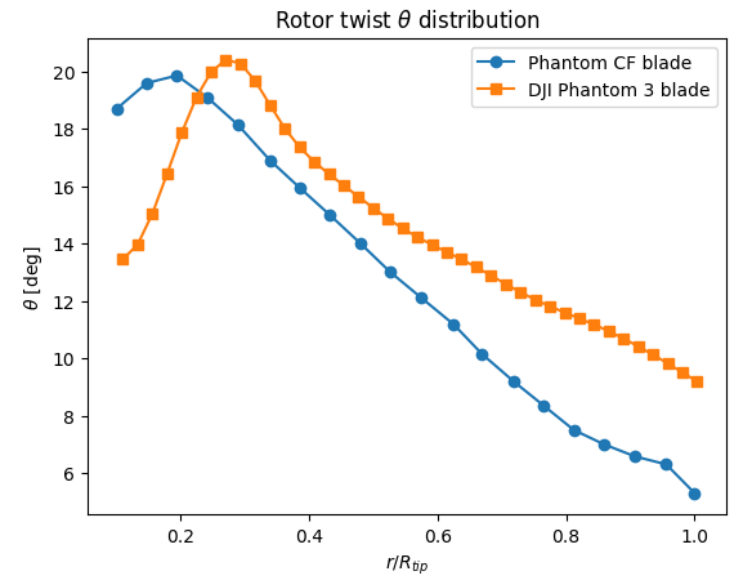

(b) Rotor twist distribution.

Figure 4. Comparison of DJI Phantom 3 factory plastic and CF blades, normalized by the rotor radius $R_{t i p}$.

The geometry for the SUI Endurance body (airframe, landing gear, canards, and camera support) has been provided by SUI to NASA Ames as a CAD model. Geometry cleaning had to be performed to remove unnecessary components and to smooth surfaces in order to obtain the reference geometry that is used in the flow solver. Once the CAD model is cleaned, it is discretized into a triangulation using a commercial software. The triangulation is imported in CGT, and the overset grids are generated.

The rotor blade geometry is the original T-Motor P15x5 CF blade. The geometry information was obtained by using high-resolution laser scanning conducted at NASA Ames. Airfoil profiles at different radii were generated from the point cloud, and the profiles were connected and smoothed, obtaining the whole blade. At the center, the blades were joined together without a hub. O-grids are used for the blades, and cap grids are generated for the blade tips. The rotor blade has a radius of $R_{t i p}=0.19 \mathrm{~m}$ and a tip chord of $c_{t i p}=0.014 \mathrm{~m}$. The length of the elongated airframe (from nose to back) is $L_{f u s}=0.43 \mathrm{~m}$, and the distance from the symmetry axis of the vehicle to the rotational axis of a rotor is $L=0.295 \mathrm{~m}$.

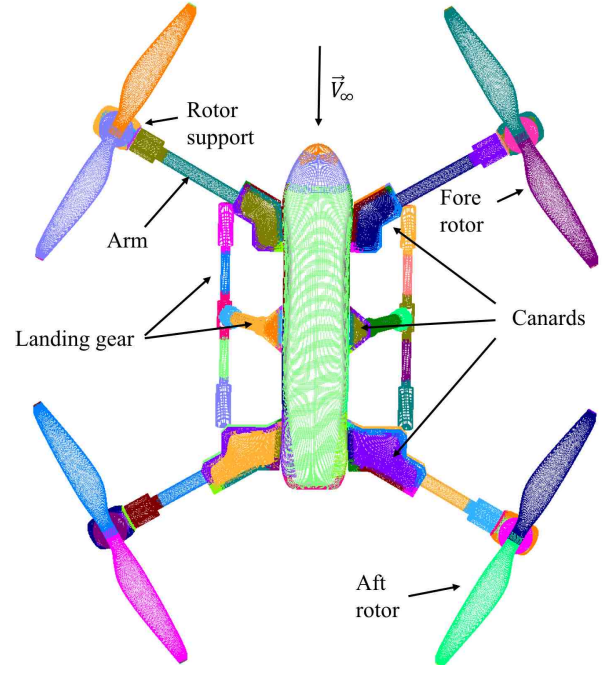

(a) SUI Endurance components.

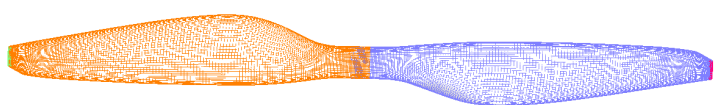

(b) SUI Endurance rotor overset surface grids.

Figure 5. Standard SUI Endurance overset surface grids. Figure (a) shows the components of the quadcopter. View from the top.

Two configurations have been studied: the standard SUI Endurance quadcopter, figure 6(a), and the hybrid configuration, figure 6(b). In the standard configuration, the four rotors are over mounted with 
respect to the arms of the airframe. In the hybrid configuration, the fore rotors are under mounted and the aft rotors are over mounted in order to get rid of the interference of the fore rotors wake with the aft rotors. To obtain the hybrid system, the grids for the fore rotors and motors are mirrored with respect to the arm's base plane, and then mirrored again to ensure $\mathrm{CW}-\mathrm{CCW}$ correct rotation.

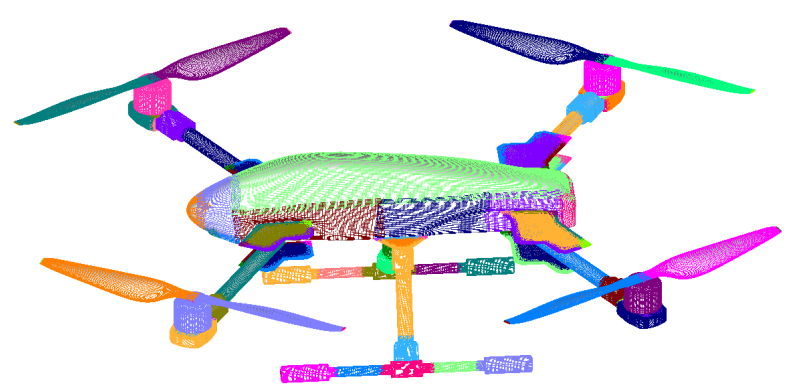

(a) Standard SUI Endurance overset surface grids.

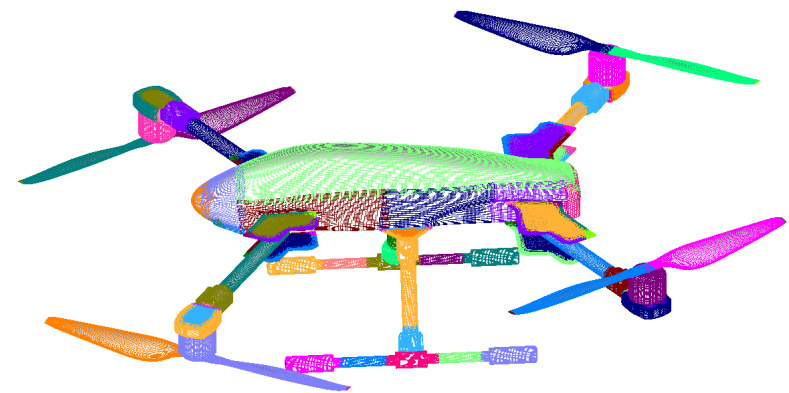

(b) Hybrid SUI Endurance overset surface grids.

Figure 6. Comparison between the SUI standard and the SUI hybrid configurations.

The resulting near-body grids for the complete SUI Endurance quadcopter, for both the standard and hybrid cases, consists of 176 overset grids, with 41 million grid points. The complete grid system off-body and near-body grids consists of 541 million grid points.

\section{Elytron $4 S U A V$ overset grids}

The last vehicle for this study is the Elytron $4 \mathrm{~S} \mathrm{UAV}$. The Elytron design combines three sets of wings: a single tilt-wing in central position with the prop-rotors mounted on it and two pairs of fixed wings. The fixed wings are split into a forward pair and an aft pair that are joined by winglets, which make use of the joined-wing concept. By splitting the wings apart, the design tries to reduce any interference with the thrust of the prop-rotors. The counter-rotating prop-rotors allow for torque cancellation. The nose fan is used for pitch control during take-off and landing.

The geometries for the Elytron $4 \mathrm{~S} \mathrm{UAV}$, its prop-rotors, and the nose fan have been provided by Elytron Aircraft LLC to NASA Ames as a STL CAD triangulation. It can be imported directly into CGT, and the overset grids are then generated.

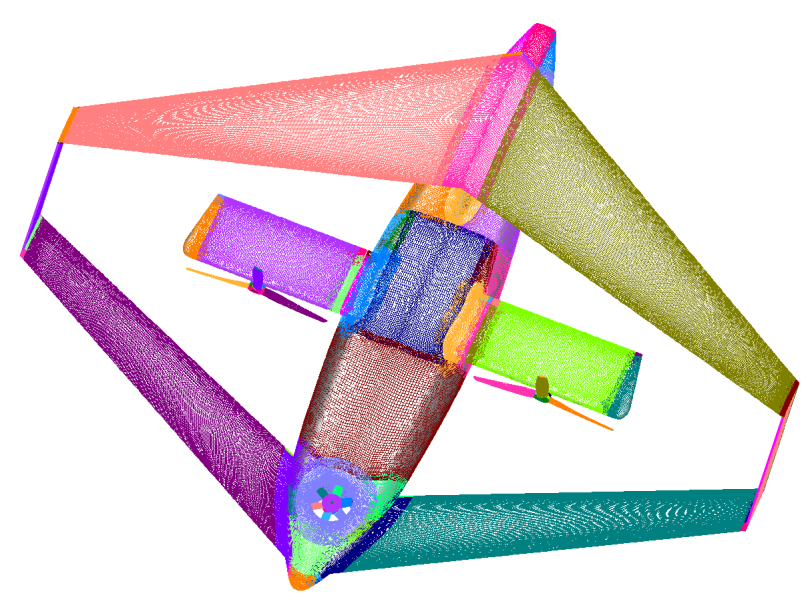

(a) Elytron overset surface grids.

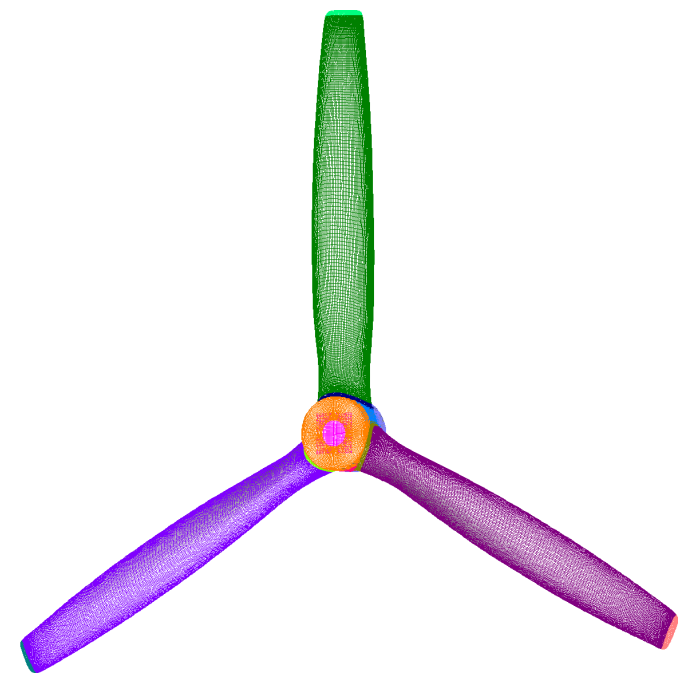

(b) Elytron prop-rotor overset surface grids.

Figure 7. Elytron 4S UAV overset surface grids. 
The Elytron prop-rotor grid system, figure 7(b), consists of three blades attached to a central hub. Ogrids are used for the blades. Cap grids are generated for the blade tips and the hub axis. The rotor blade has a radius of $R_{t i p}=0.18 \mathrm{~m}$ and a tip chord of $c_{t i p}=0.016 \mathrm{~m}$ approximately. The nose fan grids, figure 8 , consist of six blades attached to a hub. O-grids are used for the blades. Cap grids are generated for the blade tips and the hub ends. In the blade-hub junctions, collar grids are employed. The nose-fan blades have a radius of $R_{t i p}=0.04 \mathrm{~m}$ and a tip chord of $c_{t i p}=0.016 \mathrm{~m}$. In the wing-fuselage junctions and wing-winglet, collar grids are employed. The wingspan for the fixed wings is $b_{f i x}=1.66 \mathrm{~m}$, the wingspan for the tilt wing is $b_{\text {tilt }}=1.0 \mathrm{~m}$, and the length of the tear drop fuselage is $L=1.35 \mathrm{~m}$.

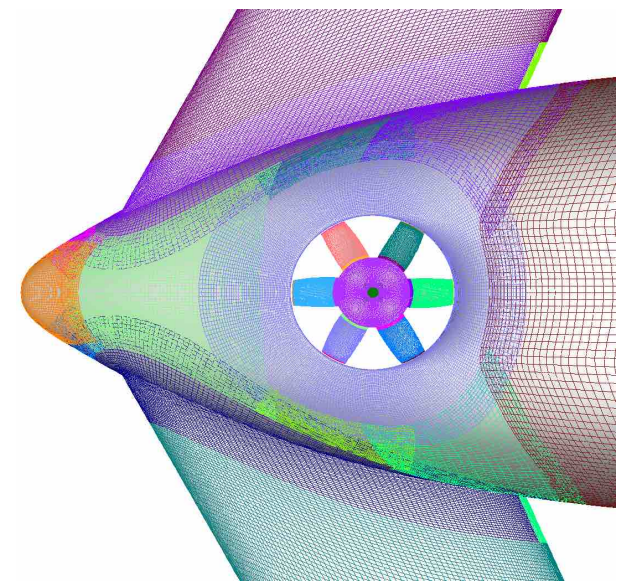

(a) Elytron nose fan view from the top.

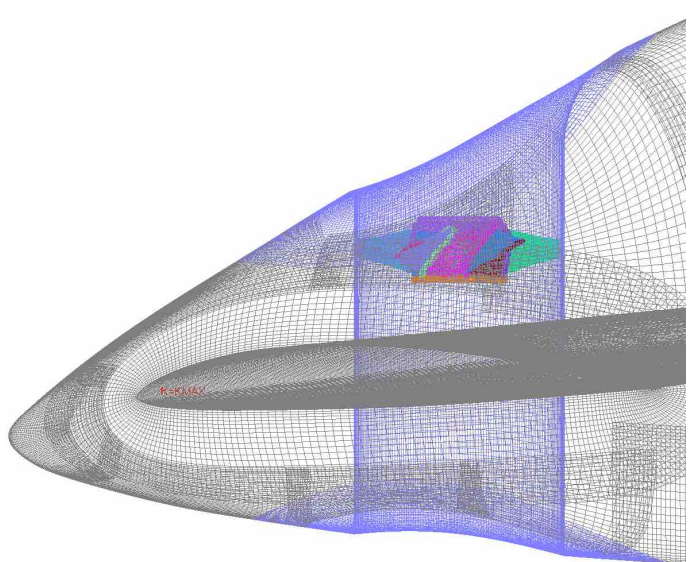

(b) Elytron nose fan view from the side.

Figure 8. Elytron $4 \mathrm{~S}$ UAV nose fan overset surface grids.

The resulting near-body grids for the complete Elytron 4S UAV consists of 147 overset grids, with a total of 361 million gris points.

\begin{tabular}{|c|c|c|c|}
\hline & DJI Phantom 3 & SUI Endurance & Elytron 4S UAV \\
\hline$R_{t i p}$ & $0.12 \mathrm{~m}$ & $0.19 \mathrm{~m}$ & $0.18 \mathrm{~m}$ \\
\hline$c_{t i p}$ & $0.010 \mathrm{~m}$ & $0.014 \mathrm{~m}$ & $0.016 \mathrm{~m}$ \\
\hline Grid points & $396 \mathrm{M}$ & $541 \mathrm{M}$ & $361 \mathrm{M}$ \\
\hline
\end{tabular}

Table 1. Geometric characteristics and number of grid points in the computational domain for the complete DJI Phantom 3, SUI Endurance and Elytron 4S UAV.

\section{B. High-Order Accurate Navier-Stokes Solver}

The Navier-Stokes equations can be solved using finite differences with a variety of numerical algorithms and turbulence models. In this study, the diagonal central difference algorithm is used with the $4^{\text {th }}$-order accurate spatial differencing option with matrix dissipation or $5^{\text {th }}$ order with scalar dissipation. The physical time step corresponds to 0.25 degrees rotor rotation, together with up to 50 dual-time sub-iterations for a 2.5 to 3.0 orders of magnitude drop in sub-iteration residual. This numerical approach and time step was previously validated for various rotor flows. ${ }^{9-12}$ In order to reduce the computational time required for a converged solution, the first 1440 steps employ a time step of $\Delta t=2.5^{\circ}$, yielding 10 rotor revolutions. The time step is then reduced to $\Delta t=0.25^{\circ}$, for which 1440 steps correspond to one rotor revolution.

\section{Low Mach Number Preconditioning}

One of the challenges for compressible Navier-Stokes methods in computing small-rotor flows is the relatively low Mach number due to small rotor radii. For example, in the case of the DJI Phantom, the Mach number at the tip of blades is under 0.2 at $5400 R P M$. Mach numbers at the inboard locations are even lower. 
Compressible Navier-Stokes codes in general suffer from slow convergence for low speed flows because of a disparity between the acoustic and convective speeds. Because most numerical algorithms have a stability restriction on the size of the time step determined by the maximum eigenvalue, the acoustic speed limits the time step. On the other hand, convergence to a steady state is controlled by the convective speed, which determines how fast low-frequency errors are advected out of the computational domain. If the convective speed is much smaller than the speed of sound, the stability restriction forces time steps so small that convergence requires a large number of iterations. Low Mach number preconditioning ${ }^{13,14}$ is an attempt to equilibrate the eigenvalues, making them all of the same order of magnitude and thus decreasing the number of iterations to convergence.

Low Mach number preconditioning is only used during the sub-iteration steps at each physical time step.

\section{Hybrid turbulence modeling}

The OVERFLOW code has a choice of algebraic, one-equation, and two-equation turbulence models, ${ }^{3}$ including hybrid RANS/LES (Large Eddy Simulation) models that close the Reynolds-Averaged Navier-Stokes equations.

The RANS equations require a closure by modeling the Reynolds stress. The one-equation SpalartAllmaras RANS turbulence model is one of the models commonly used to compute the turbulent eddy viscosity $\mu_{t}$ using the Boussinesq approximation to relate the Reynolds stresses $\tau_{i j}=\overline{u_{i}^{\prime} u_{j}^{\prime}}$ to a kinematic turbulent eddy viscosity, $\nu_{t}=\mu_{t} / \rho$, and the trace-free mean strain-rate tensor $S_{i j}^{*}$.

The Spalart-Allmaras model gives a transport equation for a viscosity-like variable $\tilde{\nu}$ :

$$
\frac{D \tilde{\nu}}{D t}=\underbrace{C_{b 1} \tilde{\nu}\left(\Omega+\frac{\tilde{\nu}}{\kappa^{2} d^{2}} f_{\nu 2}\right)}_{\text {Production }}-\underbrace{C_{w 1} f_{w}\left(\frac{\tilde{\nu}}{d}\right)^{2}}_{\text {Dissipation }}+\underbrace{\frac{1}{\sigma}\left[\nabla \cdot((\nu+\tilde{\nu}) \nabla \tilde{\nu})+C_{b 2}(\nabla \tilde{\nu})^{2}\right]}_{\text {Diffusion }}
$$

With the turbulent eddy viscosity given by $\nu_{t}=\tilde{\nu} f_{\nu 1}$ and where $D() / D t$ is the material time derivative.

The right-hand side of Eq. (1) consists of turbulence production, dissipation, and diffusion source terms. The constants $C_{b 1}, C_{b 2}, C_{w 1}, \kappa$ and functions $f_{\nu 1}, f_{\nu 2}$ and $f_{w}$ are described by Spalart and Allmaras. ${ }^{15}$ The damping function $f_{\nu 1}$ reduces $\nu_{t}$ near the wall. Note that the OVERFLOW code uses the vorticity magnitude $\Omega$ instead of the strain-rate tensor since these two variables are approximately equal in a boundary layer and the vorticity is already available in OVERFLOW.

The turbulence length scale, $d$, is defined as the distance from a field point to the nearest wall. The accuracy of the Spalart-Allmaras model depends strongly on the source terms in Eq. (1), which were primarily developed for attached boundary-layer flows along flat plates, wings, fuselages, etc.

The turbulence length scale, $d$, plays a key role in accurately determining the rotor Figure of Merit, $F M$. The $F M$ is defined as the ratio of ideal power for a rotor in hover obtained from momentum theory and the actual power consumed by the rotor. ${ }^{16}$ A problem occurs deep within the rotor wake, where $d$ may be several rotor radii in length. In this case, $d$ no longer represents an estimate of the largest turbulent eddy in the local flow but is rather a very large geometric parameter. When $d$ is very large the turbulence dissipation becomes very small. On the other hand, the strong tip vortices in the lower wake can generate significant turbulence production. Over time, this imbalance in turbulence production and dissipation in the lower wake can result in excessively large eddy viscosities. These large viscosities can migrate up the vortex wake after several rotor revolutions and, under blade-vortex interaction conditions, infiltrate the blade boundary layers. When this happens, the rotor blade drag and torque increase significantly and artificially, resulting in large $F M$ errors and an under-prediction of rotor efficiency.

An additional degree of realism can be obtained by the use of Large Eddy Simulation (LES). In LES, the large turbulent scales are resolved using a small grid spacing $\Delta$, and the small scales are modeled. A low-pass spatial filter is applied to the Navier-Stokes equations, associated with a cut-off length. Below the cut-off length the subgrid-scales (SGS) must be modeled. An implication of Kolmogorov's theory of self similarity is that the large eddies of the flow are dependent on the geometry while the smaller scales more universal. This feature allows one to explicitly solve for the large eddies in a calculation and implicitly account for the small eddies by using a SGS model. Smagorinsky ${ }^{17}$ first postulated a SGS model for the Reynolds stresses based on:

$$
\tau_{i j}=2 \nu_{t} S_{i j}
$$




$$
S_{i j}=\frac{1}{2}\left(\frac{\partial U_{i}}{\partial x_{j}}+\frac{\partial U_{j}}{\partial x_{i}}\right)
$$

where $S_{i j}$ is the resolved strain-rate tensor and $\nu_{t}$ is the Smagorinsky eddy viscosity given by:

$$
\nu_{t}=\left(C_{s} \Delta\right)^{2} \sqrt{S_{i j} S_{i j}}
$$

and $C_{s}$ is the Smagorinsky coefficient. The filter width $\Delta=(\Delta x \Delta y \Delta z)^{1 / 3}$ is chosen to be the geometric mean of the grid cell spacing.

The use of LES throughout the computational domain is impractical for the Reynolds numbers found in common rotor flows. This is due to the very small length scales of wall-bounded flows.

The Detached Eddy Simulation (DES) model $^{15}$ is a more practical alternative. The intent of DES is to be, in effect, a RANS model throughout the boundary layer, where the turbulent scales can be very small and need to be modeled via RANS, and an LES model outside the boundary layer where the largest turbulent scales are grid-resolved. In this way, DES is a RANS/LES hybrid approach that mitigates the problem of artificially large eddy viscosity. The turbulence length scale $d$ is replaced by $\bar{d}$ as given by Eq. (5); $\bar{d}$ is the minimum of the distance from the wall $d$ and the local grid spacing $\Delta=\max (\Delta x, \Delta y, \Delta z)$ times a coefficient $C_{D E S}$.

Therefore, the Detached Eddy Simulation with the Spalart-Allmaras model transport equation for $\tilde{\nu}$ is:

$$
\frac{D \tilde{\nu}}{D t}=\underbrace{C_{b 1} \tilde{\nu}\left(\Omega+\frac{\tilde{\nu}}{\kappa^{2} \bar{d}^{2}} f_{\nu 2}\right)}_{\text {Production }}-\underbrace{C_{w 1} f_{w}\left(\frac{\tilde{\nu}}{\bar{d}}\right)^{2}}_{\text {Dissipation }}+\underbrace{\frac{1}{\sigma}\left[\nabla \cdot((\nu+\tilde{\nu}) \nabla \tilde{\nu})+C_{b 2}(\nabla \tilde{\nu})^{2}\right]}_{\text {Diffusion }}
$$

with:

$$
\bar{d}=\min \left(d, C_{D E S} \Delta\right)
$$

The fixed coefficient is given by $C_{D E S}=0.65$. This simple but crucial change can be viewed in two different ways. From a numerical perspective the length scale has been significantly reduced. This allows the turbulence dissipation to remain active in the vortex wake below the rotor plane and prevents the turbulent eddy viscosity from growing to unrealistic values. The torque therefore remains unaffected, compared to the process described above, and the $F M$ is accurately predicted. A physical interpretation views the modified length scale as an implicit filter, where the largest turbulent eddies are now grid-resolved. All smaller eddies are modeled by a reduced turbulent eddy viscosity. This DES approach provides a rational way to reduce the length scale, and hence the turbulent eddy viscosity, based on a physical model.

The DES approach assumes that the wall-parallel grid spacing $\Delta_{\|}$exceeds the thickness of the boundary layer $\delta$ so that the RANS model remains active near solid surfaces. If the wall-parallel grid spacing is smaller than the boundary layer thickness, $\Delta_{\|}<\delta$, then the DES Reynolds stresses can become under-resolved within the boundary layer, and this may lead to non-physical results, including grid-induced separation. Using Delayed Detached Eddy Simulation (DDES) ${ }^{18}$ the RANS mode is prolonged and is fully active within the boundary layer. The wall-parallel grid spacing used in this study does not violate the hybrid-LES validity condition. Thus DES and DDES should give similar results. Nevertheless, all computations have been performed using the DDES model for both NB and OB grids.

\section{Results}

The OVERFLOW Navier-Stokes CFD code is used throughout this study. All CFD computations were carried out with NASA's supercomputers Pleiades and Electra located at the NASA Advanced Supercomputer (NAS) facilities.

\section{A. DJI Phantom 3}

Single rotor

The geometry differences in chord and twist distribution between the CF blade and the originalPhantom 3 blade are highlighted in figure 4 . The Phantom CF blade has a considerably lower twist than the fabric Phantom 3 blades. At outboard radial stations where most of the thrust is generated, the difference in twist is approximately $4^{\circ}$. Therefore, the pitch angle for the CF blade is increased by $4^{\circ}$ in the simulations to 
compensate for the difference. The effects of the pitch angle on the integrated performances for small rotor blades is given by Yoon, ${ }^{12}$ where it is found that the FM peaks for a pitch angle of $8^{\circ}$. In addition, the airfoil shapes of the CF blade near the tip seem more like a thick plate than a airfoil, probably due to the manufacturing process.

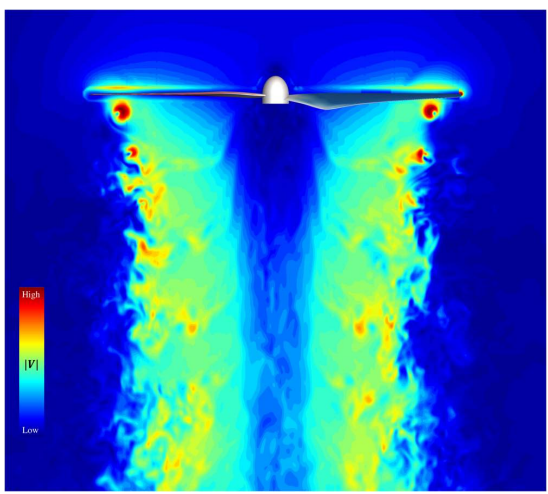

(a) DJI Phantom 3 original blade velocity magnitude.

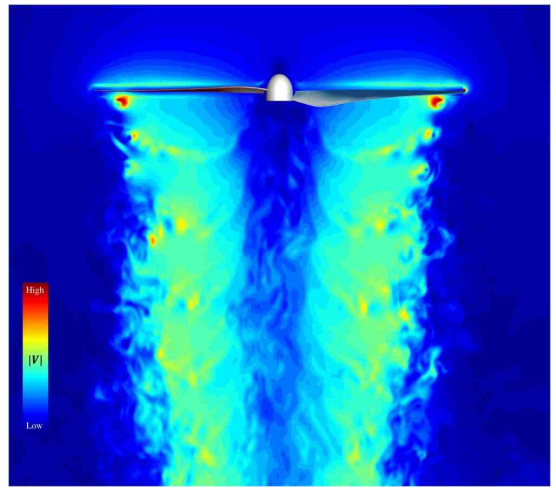

(d) CF Phantom blade velocity magnitude.

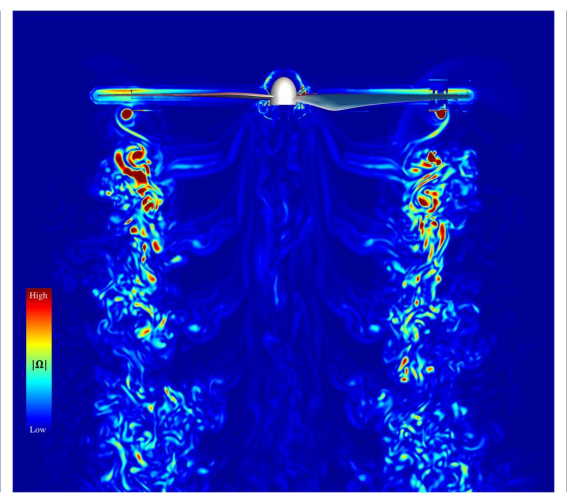

(b) DJI Phantom 3 original blade vorticity magnitude.

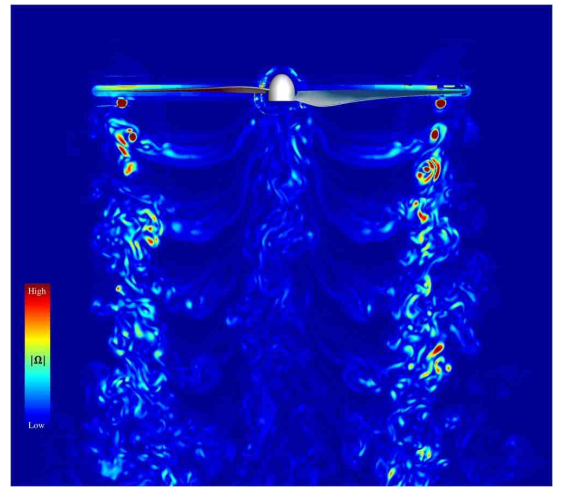

tude.

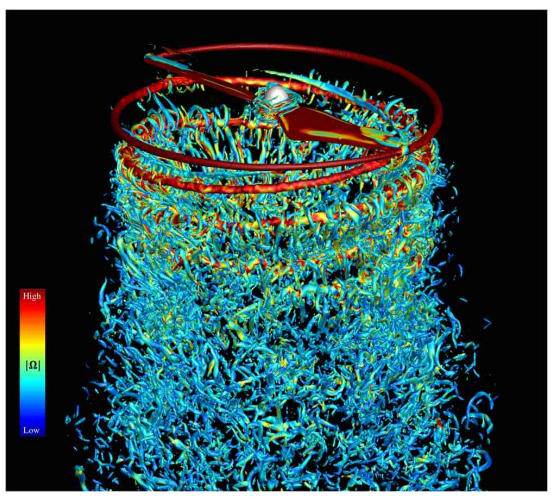

(c) DJI Phantom 3 original blade Qcriterion vorticity.

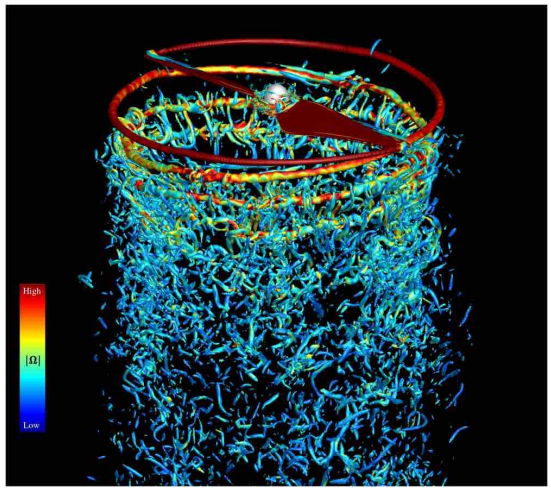

(f) CF Phantom blade Q-criterion vorticity.

Figure 9. Comparison of DJI Phantom 3 and CF blade rotor wakes. Velocity magnitude and vorticity magnitude slices at $y=0$. Q-criterion vorticity iso-surfaces colored with the vorticity magnitude. Pressure shown at the body surface: red means high pressure, blue low pressure.

Figures 9(a) and (d) show a slice of the velocity magnitude at $5400 R P M$ for the DJI Phantom 3 blades and the Phantom CF blades. High downwash speeds indicated by red and yellow suggest increases in thrust. The pressure is shown at the surface of the blade. Figures $9(\mathrm{~b})$ and (e) show a slice of the vorticity magnitude for the blades. Figures 9(c) and (f) show the Q-criterion vorticity iso-surfaces.

All of these characteristics indicate that the DJI Phantom 3 blade is a better design. In effect, the DJI Phantom 3 original blade generates $25 \%$ more thrust than the Phantom CF blades at $N_{\text {hover }}=5400 R P M$. The thrust coefficient $c_{T}$ is calculated in all cases by averaging the instantaneous $c_{T_{i}}$ over the last five revolutions. The thrust coefficient is defined as:

$$
c_{T}=\frac{T}{\frac{1}{2} \rho_{\infty} A\left(\Omega R_{t i p}\right)^{2}}
$$

where $T$ is the thrust, $\rho_{\infty}$ the density, $A$ the reference area, and $\Omega$ the rotational velocity.

\section{Quadcopter in hover}

Figure 10 compares the velocity for the three quadcopters. First, we compare the effect of a real airframe with a simplified airframe with the same rotors. The presence of the main airframe, the battery, and the camera in the first configuration, figure 10(a), reduces the interactions between rotors. Thus, the rotors for 
the complete Phantom 3 configuration generate more thrust than the simplified airframe in figure 10(b). However, the landing gear and the battery in the complete DJI Phantom 3 are immersed in the wakes of the rotors and are being pushed downward by the airflow. This generates an airframe download higher than in the case of the simplified airframe, leading finally to a total thrust reduction of $1 \%$ for the complete DJI Phantom 3. When comparing the same airframe with different rotors, figures 10 (b) and 10(c), the importance of the rotors is clear, as we find an improvement of $26 \%$ in the quadcopter thrust coefficient $c_{T}$ for the simplified Phantom 3. This agrees well with the integrated performances of the single blades. The presence of the airframe produces a download but reduces the interactions between rotors. Four rotors generate less thrust without the airframe ${ }^{12}$ than with it.

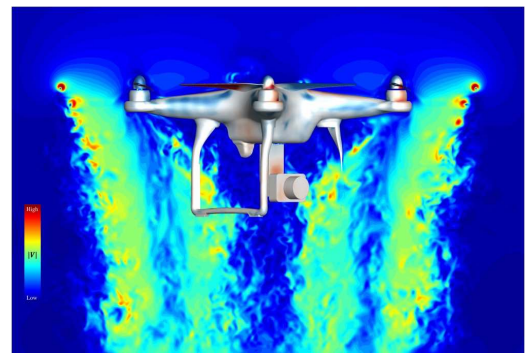

(a) DJI Phantom 3 complete.

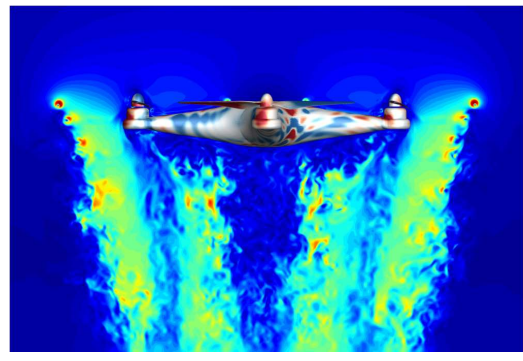

(b) Simplified Phantom 3.

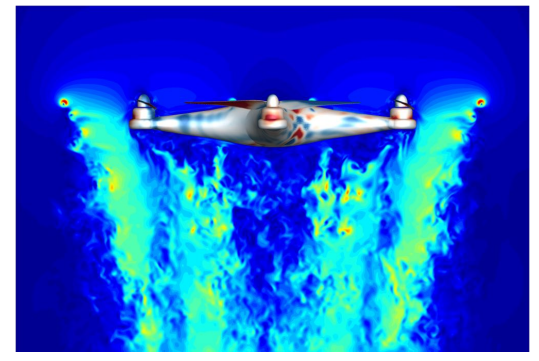

(c) Simplified CF Phantom 3.

Figure 10. DJI Phantom 3 quadcopter rotor wakes in hover. Velocity magnitude slices at $y=0$. Pressure shown at the body surface: red means high pressure, blue low pressure.

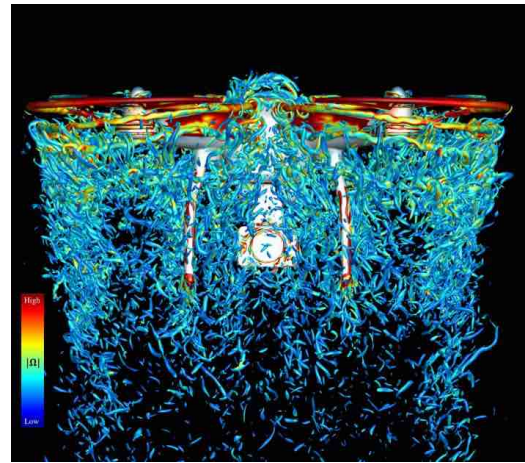

(a) DJI Phantom 3 complete.

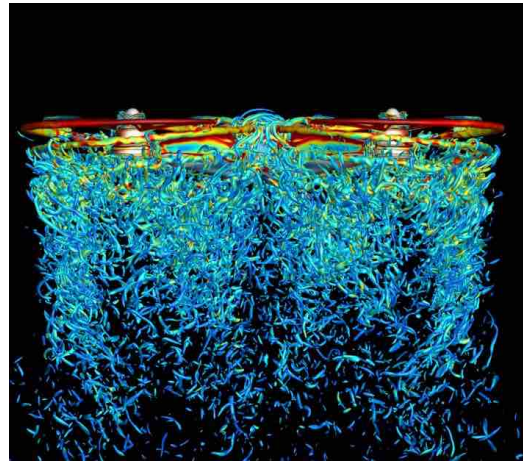

(b) Simplified Phantom 3.

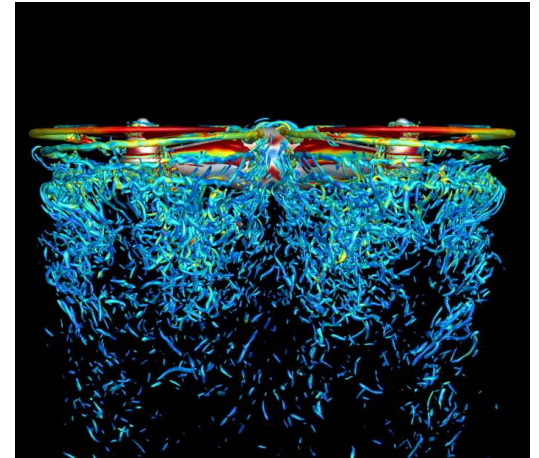

(c) Simplified CF Phantom 3.

Figure 11. Front view DJI Phantom 3 quadcopter in hover. Q-criterion vorticity iso-surfaces colored with the vorticity magnitude.

Figure 11 shows the Q-criterion vorticity iso-surfaces colored by the vorticity magnitude for the three configurations. The images show the complex 3D structures of the flow due to aerodynamic interactions. Besides the blade-tip vortices, in the front and back two vortices are created as reverse flow comes from below the quadcopter to answer the need of more air to feed the rotors. These vortices are stronger when more components are added to the airframe; see figure 14 for a view from the top. The development of the wake is weaker for the simplified CF Phantom 3.

The integrated performances for the three configurations are shown in figure 12. The thrust coefficient is normalized by four times the thrust coefficient of the single rotor DJI Phantom 3. As mentioned in the previous paragraphs, the airframe increases the thrust of the rotors because it reduces interactions, and it also acts as ground effect. We can observe this effect in the chart of figure 12, where the $c_{T}$ for the complete DJI Phantom 3 and the simplified Phantom 3 is above 1. However, the rotor wakes push down the airframe, generating a download (negative thrust) and therefore reducing the total thrust of the quadcopter. The download is higher for the complete DJI Phantom 3 than for the simplified Phantom 3. This results in a $1 \%$ thrust reduction for the complete configuration compared to the simplified Phantom 3. The simplified configuration produces $26 \%$ more thrust than the simplified CF configuration. The effect of the camera on the integrated performances is negligible. However, further down in this section we will see that the presence 


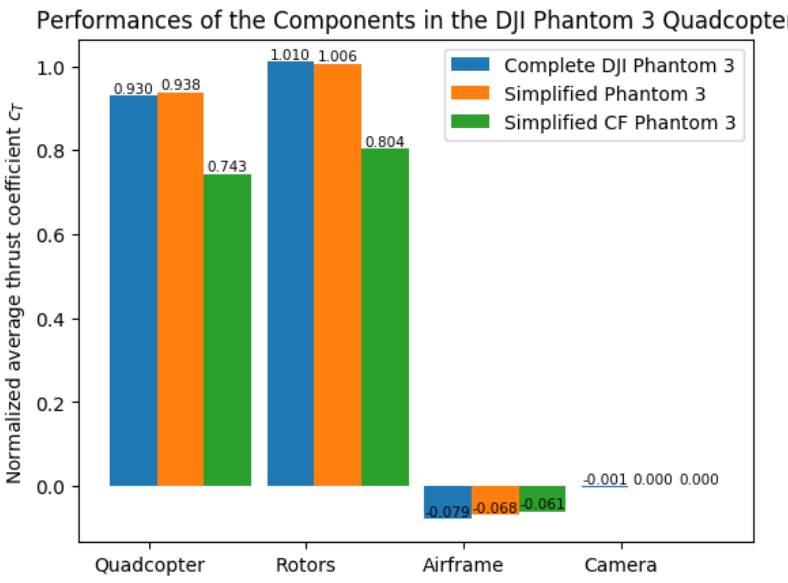

Figure 12. Normalized average thrust coefficient for the components in the different Phantom 3 configurations. The thrust coefficient is averaged over five revolutions and normalized by four times the thrust coefficient of the single rotor DJI Phantom 3 original.

of the camera and battery block the propagation of pressure waves, reducing the noise of the configuration.

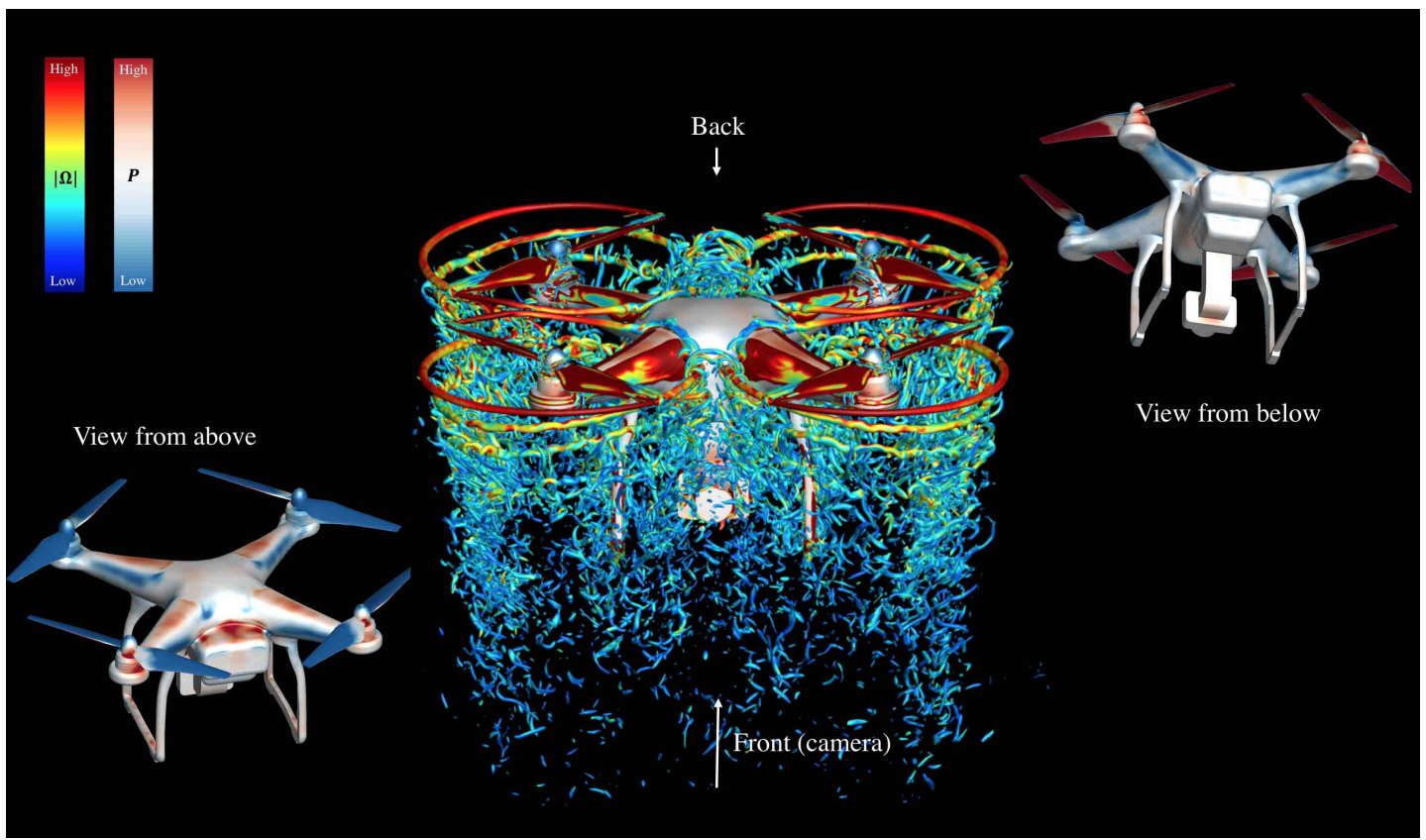

Figure 13. Oblique view of the complete DJI Phantom 3 in hover. Q-criterion vorticity iso-surfaces colored with the vorticity magnitude. Pressure is shown at the body surface: red means high pressure, blue low pressure.

Figure 13 shows in detail the pressure on the surface of the complete quadcopter. We have seen that the rotors produce more thrust in the configuration with components underneath the airframe because the components reduce the rotor-rotor interactions. But some of these components are immersed in the wakes, thereby being pushed down. This can be observed in figure 13 on the left of the image, where the battery and landing gear exhibit wide zones in red.

To conclude this section, figure 14 shows the top view of Q-criterion vorticity iso-surfaces for the complete DJI Phantom 3 and the simplified Phantom 3 colored by the pressure. The interactions between rotors are stronger in the simplified Phantom 3 configuration as no components are added in the bottom of the 
fuselage that can block these interactions. With stronger interactions, the pressure fluctuations are stronger and more frequent, as observed in the images. Low-fidelity CFD codes need to run a separate computational aeroacoustics solver (CAA) in order to calculate the aeroacoustic fields. However, recent studies by Nicolas Zawodny (to be published) have shown that a high-fidelity solver such as OVERFLOW predicts the pressure fluctuations correctly, at least qualitatively. With this, one can conclude that the simplified Phantom 3 configuration is probably noisier than the complete DJI Phantom 3 configuration.

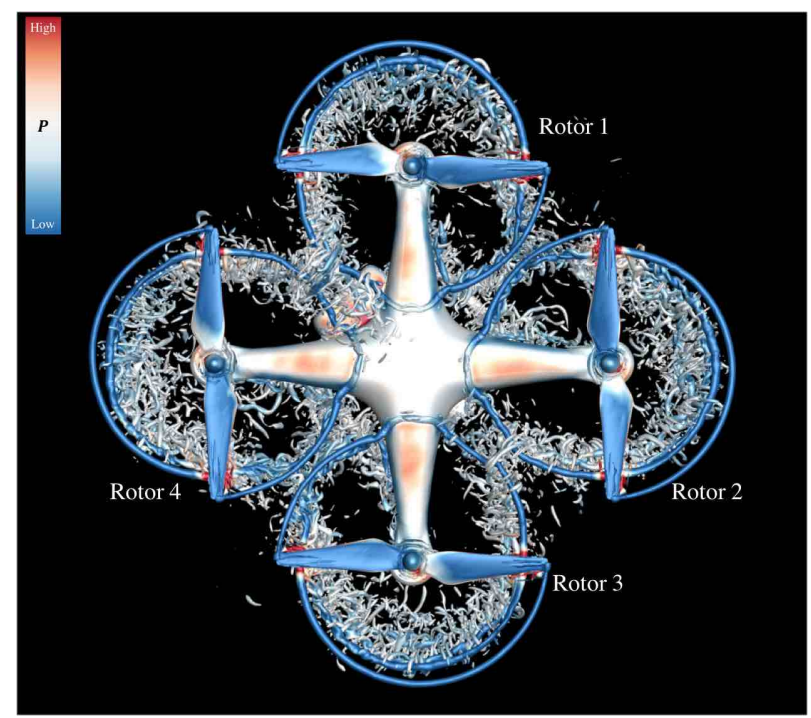

(a) DJI Phantom 3 complete.

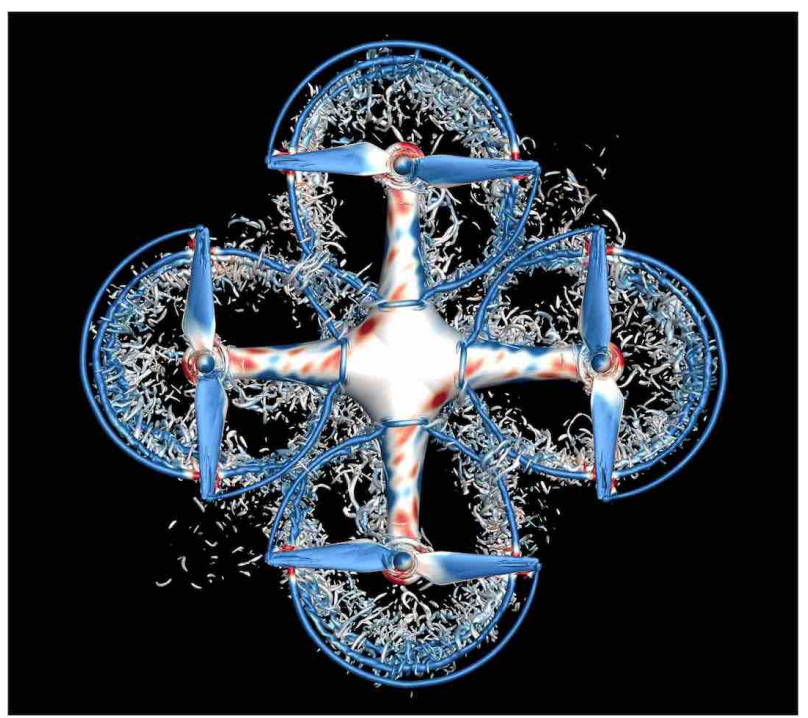

(b) Simplified Phantom 3.

Figure 14. Top view of DJI Phantom 3 quadcopter in hover. Q-criterion vorticity iso-surfaces colored with the pressure. Pressure shown at the body surface: red means high pressure, blue low pressure. The simplified Phantom 3 configuration is noisier.

\section{B. Weather effects on quadcopters-Wind gusts}

Small multi-rotor UAVs have poor stability in gusts due to the generation of a strong pitching moment when a sudden wind impacts the UAV. For the first time, a complete quadcopter has been simulated under the effect of weather such as wind gusts. Previous studies of wind gust experiments on quadrotors showed that the use of slant rotors reduces the pitching moment significantly. ${ }^{19}$ In this study, the complete DJI Phantom 3 during hover has been simulated with wind gusts at different wind velocities $V_{\text {wind }}$ and sideslip angles $\beta$. Here we suppose that the gust impacts the quadcopter after the solution for hover is converged, i.e. after 30 revolutions. We model the gust as a constant velocity in the horizontal plane. The effect of wind gusts is measured by the moment produced. This moment is the consequence of a difference in thrust between rotors.

Different wind velocities are simulated. We have limited the maximum wind speed to $25 \mathrm{kts}$ because, according to the Beaufort wind force scale, a Beaufort number of 6 (22 to $27 \mathrm{knots}$ ) is equivalent to a strong breeze, in which umbrellas are used with difficulty.

Figure 15 gives a view from the top and shows how the wakes are affected due to the incoming gust for different angles $\beta$ and wind velocities. Naturally, as the velocity increases the effect on the wakes becomes stronger and the rotor-rotor interactions are more important. With increasing velocities, the supertip vortices are stronger and thus easier to see. The asymmetries are also noticeable, see for example (g) and (h) where some vortices are even on top of the airframe. For $\beta=0^{\circ}$, the wind gust first impacts rotor 4; see images (a), (b) and (c). For $\beta=45^{\circ}$ the gusts come from the back, where the battery is placed, along the bisecting line between rotors 1 and 4 , see pictures (d) and (e). For the last group of simulations, the gusts have an angle of $\beta=135^{\circ}$, and they impact the quadcopter on the side, where the camera is; see pictures (f), $(\mathrm{g})$ and (h).

Figure 16 shows the thrust coefficient $c_{T}$ of each rotor and the moments as a function of the wind angle and velocity. The thrust difference between opposed rotor generates a moment. The thrust increases with 


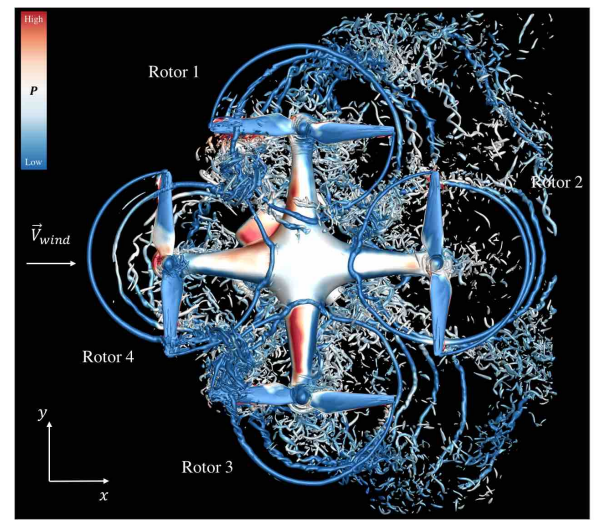

(a) $V_{\text {wind }}=10 \mathrm{kts}, \beta=0^{\circ}$.

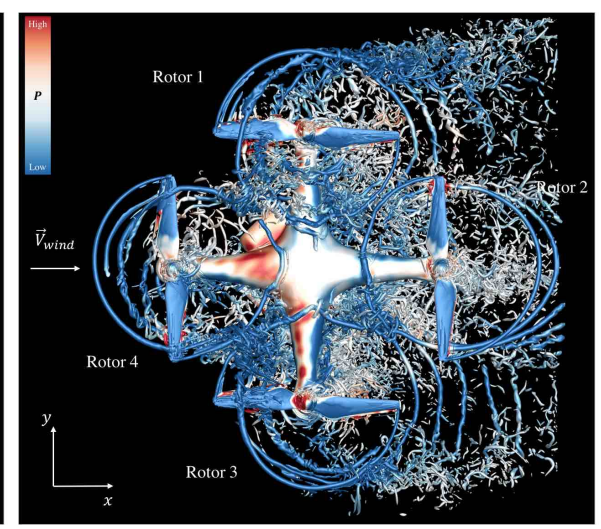

(b) $V_{\text {wind }}=17 \mathrm{kts}, \beta=0^{\circ}$.

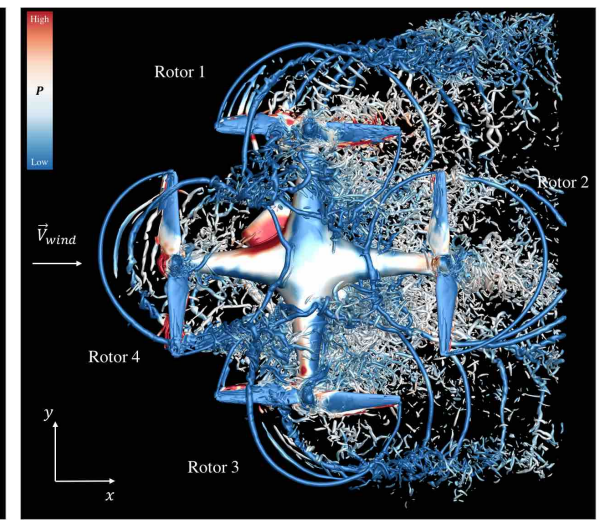

(c) $V_{\text {wind }}=20 \mathrm{kts}, \beta=0^{\circ}$.

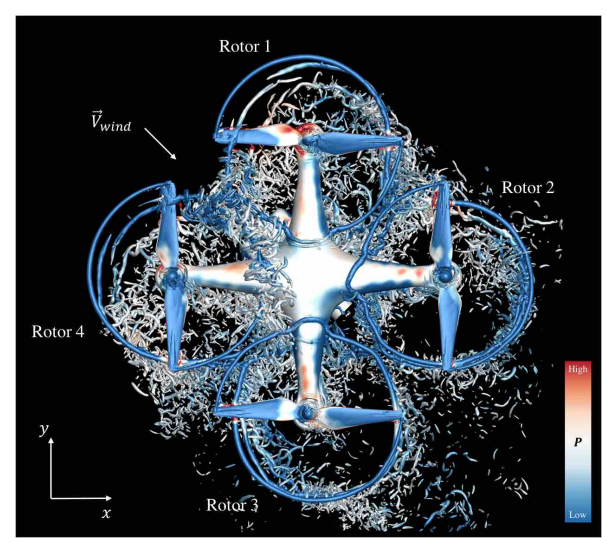

(d) $V_{\text {wind }}=10 \mathrm{kts}, \beta=45^{\circ}$.

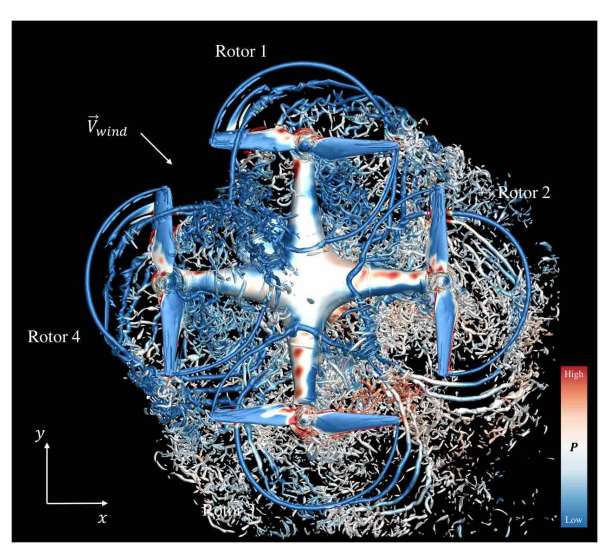

(e) $V_{\text {wind }}=20 \mathrm{kts}, \beta=45^{\circ}$.

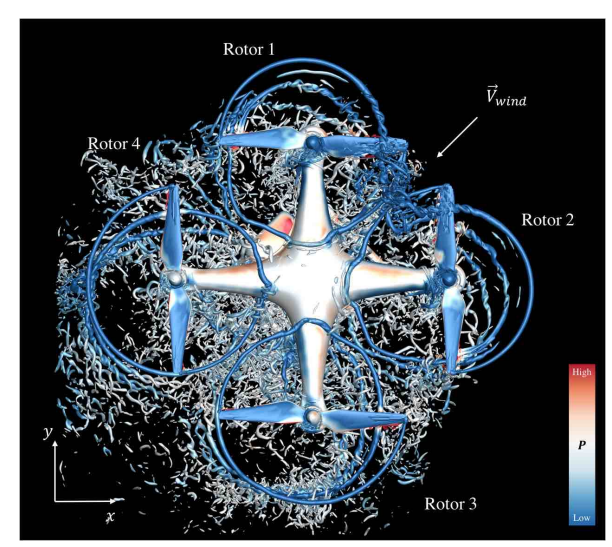

(f) $V_{\text {wind }}=10 \mathrm{kts}, \beta=135^{\circ}$.

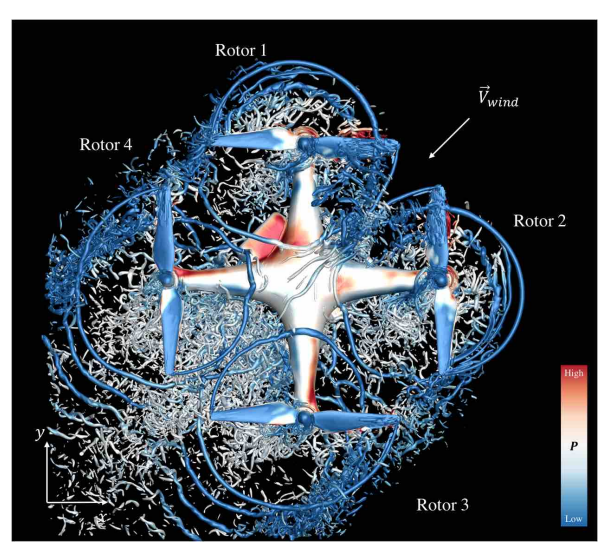

(g) $V_{\text {wind }}=17 \mathrm{kts}, \beta=135^{\circ}$.

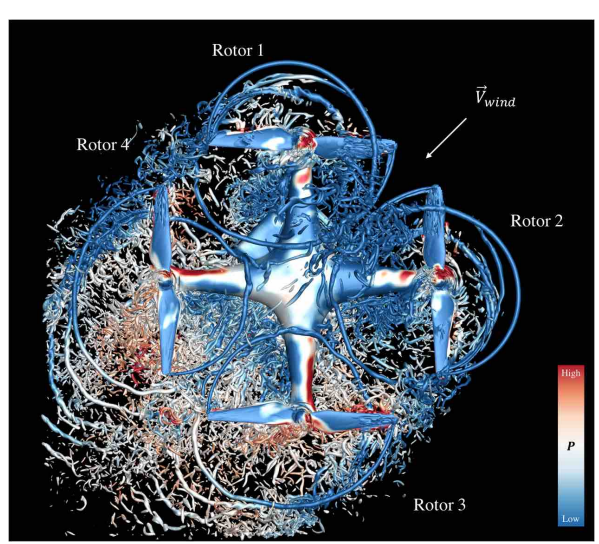

(h) $V_{\text {wind }}=25 \mathrm{kts}, \beta=135^{\circ}$.

Figure 15. DJI Phantom 3 quadcopter under the effect of wind gusts, for an incoming wind gust through a rotor, $\beta=0^{\circ}$, from the back, $\beta=45^{\circ}$ and from the side, $\beta=135^{\circ}$. 


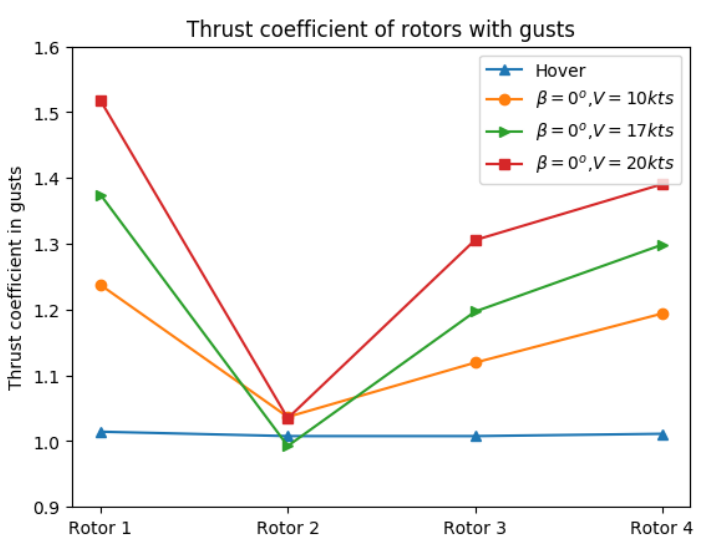

(a) Coefficient of thrust $c_{T}$ of each rotor, $\beta=0^{\circ}$.

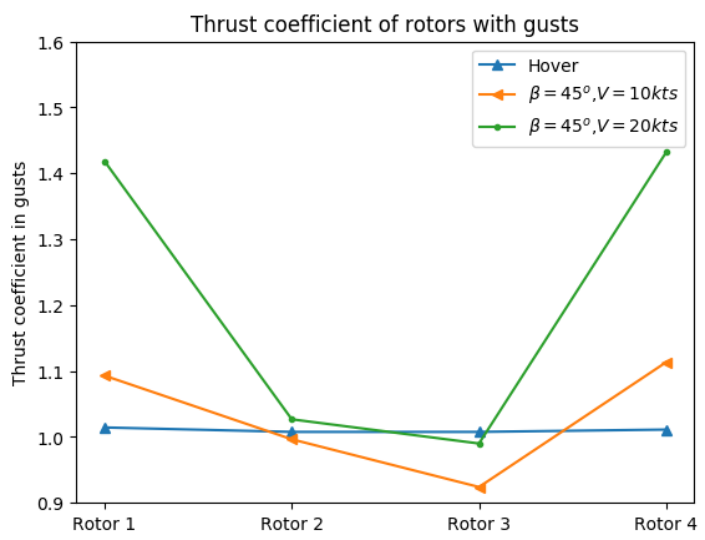

(c) Coefficient of thrust $c_{T}$ of each rotor, $\beta=45^{\circ}$.

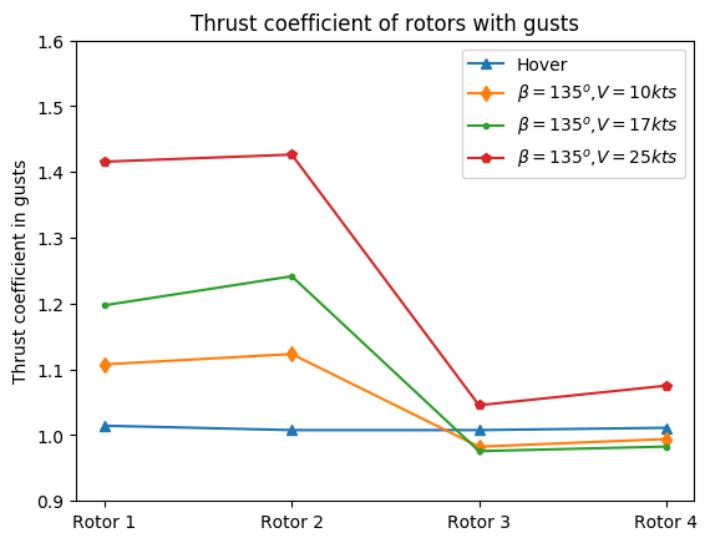

(e) Coefficient of thrust $c_{T}$ of each rotor, $\beta=135^{\circ}$.

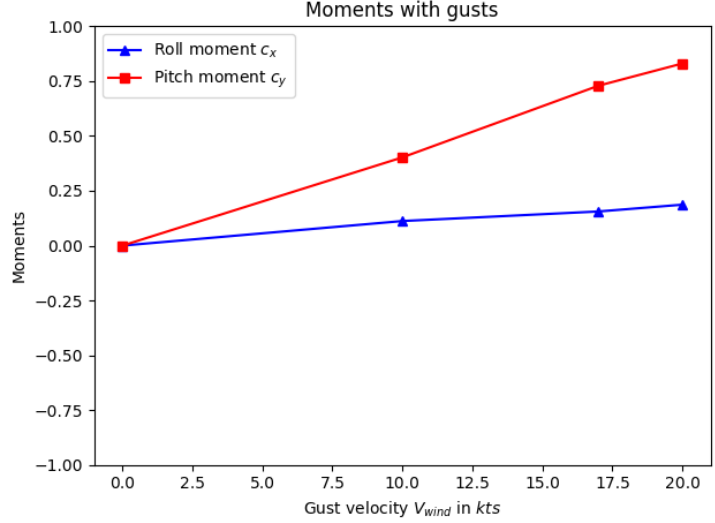

(b) Moments $c_{x}$ and $c_{y}, \beta=0^{\circ}$.

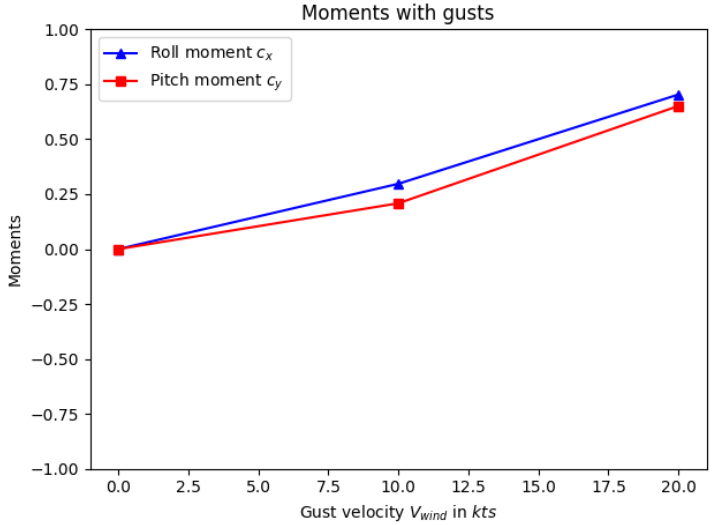

(d) Moments $c_{x}$ and $c_{y}, \beta=45^{\circ}$.

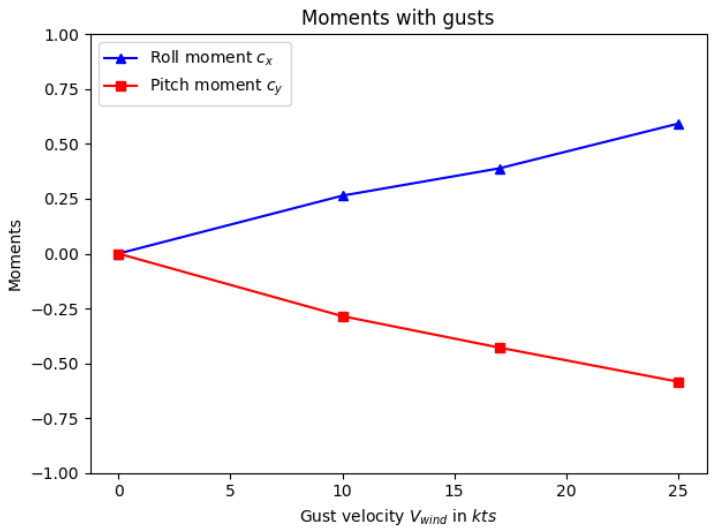

(f) Moments $c_{x}$ and $c_{y}, \beta=135^{\circ}$.

Figure 16. Effects of gusts on the quadcopter for an incoming wind gust through a rotor, $\beta=0^{\circ}$, from the back, $\beta=45^{\circ}$ and from the side, $\beta=135^{\circ}$. The thrust coefficient is nondimensionalized by the thrust coefficient of a single rotor. The moments are nondimensionalized by the thrust coefficient of a single rotor times the length of the arm. 
the wind velocity and so do the thrust differences between rotors. Therefore, the total moment is stronger. If the moment is not compensated by spinning the rotors that generate less thrust faster (by using the $\mathrm{ESC}^{\mathrm{d}}$ ), the moment will eventually flip the quadcopter, destabilizing it and probably making it crash. If the wind velocity is too strong, the controls may not act quickly enough. This result raises the importance of understanding and quantifying the effects of weather on small quadcopters and also in larger multi-rotor vehicles such as Urban Air Mobility. In order for UAM to be viable, the vehicles must be robust enough to be able to safely overfly our cities.

\section{SUI Endurance}

The SUI endurance design is improved for forward flight. The airplane-like fuselage and the canards have low drag and contribute to the lift in forward flight, even for small negative angles of attack. Quadcopters, like helicopters, need to have a negative angle of attack $A o A<0$ in order to generate forward thrust. With a negative $A o A$ the rotor disk plane is leaning with a horizontal component generating a forward thrust to overcome the drag and a vertical component contributing to the lift in order to balance the weight.

Rotor-rotor interactions are smaller in hover than those for the DJI configuration. However, in forward flight, as the forward velocity increases, rotor-rotor interactions are stronger because the wakes of the fore rotors begin to affect the aft rotors, harming their performance. For clarity, from now on the thrust produced by the rotors will be decomposed into its two components: forward thrust and lift. Again, all force coefficients have been averaged for the last five revolutions. They are nondimensionalized by the thrust produced by a single SUI rotor in hover, $N_{\text {hover }}=3600 R P M$.

In this study, the SUI Endurance is simulated in forward flight for a flow velocity of $V_{\infty}=10 \mathrm{~m} / \mathrm{s}$ $(22.37 \mathrm{mph})$ with an angle of attack of $A o A=-7.7^{\circ}$; see figure 17 . Conditions for trim were measured in flight tests. Fore and aft rotors rotate at different rotational speeds for the quasi-steady forward flight condition: the fore rotors rotate at $N=3510 R P M$ and the aft rotors rotate at $N=4410 R P M$; that is, the aft rotors rotate $25.6 \%$ faster than the fore rotors. The advance ratio based on the fore rotors is 0.1429. Rotor-rotor interactions are strong as the wakes of the fore rotors impact the aft rotors. Supertip vortices from both fore and aft rotors are visible. The inboard supertip vortices from the fore rotors interact with the fuselage and then are fed into the advancing side of the aft rotors near the juncture of the fuselage and the aft canards. There are some blade-vortex interactions in the fore rotors. The aft rotors generate approximately $30 \%$ higher lift than the fore rotors. However, the aft rotors performances could be better if they were not immersed in the wake from the fore rotors.

With the idea of increasing the performance of the aft rotors, the authors decided to under-mount the fore rotors and keep the aft rotors over-mounted, to obtain what we have called a hybrid configuration, see figure 18 for the Q-criterion vorticity iso-surfaces colored by the pressure. With this configuration, the aft rotors are no longer immersed in the wake of the fore rotors; thus in theory they should generate more thrust for the same conditions. For this reason, the hybrid configuration is a much better design: while the lift is slightly reduced because of the under-mount rotors, ${ }^{12}$ the forward thrust is increased by $63 \%$ compared to the standard configuration.

The charts in figure 19 show in detail the performances of the quadcopter and of each component. Figure 20 shows the forces acting on the quadcopter. Taking a closer look to the performances in figure 19, the total lift coefficient is $0.8 \%$ higher for the standard quadcopter. The fore rotors' lift is slightly reduced in the hybrid configuration, but the lift of the aft rotors is increased almost $6 \%$. The aft rotors in the hybrid configuration generate $40 \%$ more lift, while in the standard design they generate $30 \%$ more lift. As we described in a previous paragraph, the aft rotors performances are improved if they interact less with the fore rotors. In the hybrid configuration, the fuselage and the canards generate less lift, and the negative lift from the arms is more important.

The big difference in the performances comes when comparing the horizontal forces. In the hybrid configuration, the fore rotors generate $5 \%$ more thrust and the aft rotors generate $6 \%$ more thrust than the standard configuration. Moreover, the drag from the arms is greatly reduced, with $22 \%$ less drag than in the standard configuration. This big reduction is due to the fact that the arms are no longer in the wake of the fore rotors. The flow around the arms has therefore a smaller velocity and the drag is reduced. The landing gear generates more drag because it is closer to the fore rotors. When adding all the forces, there is an improvement of $63 \%$ in the forward thrust for the hybrid configuration.

\footnotetext{
${ }^{\mathrm{d}}$ Electronic Speed Control
} 


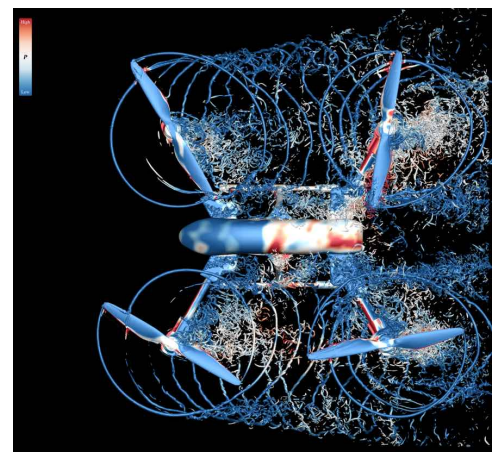

(a) Top view

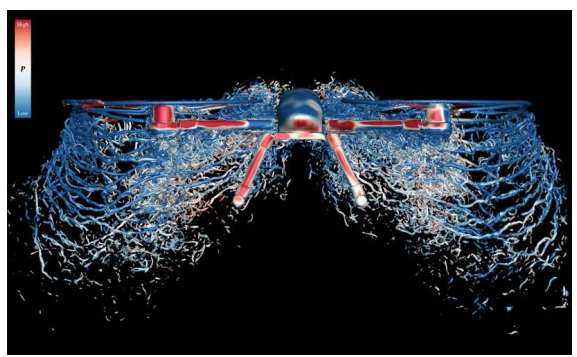

(c) Front view

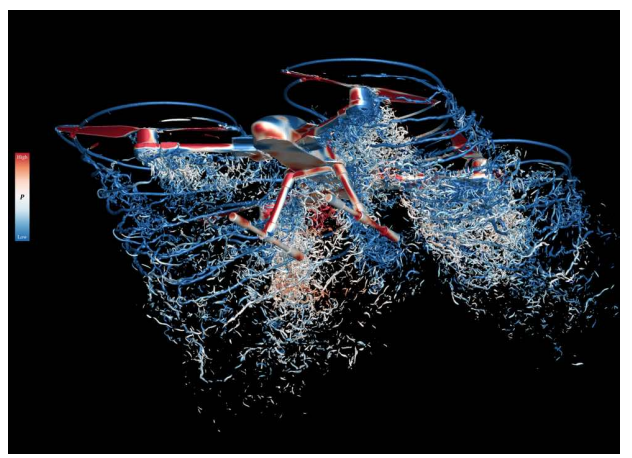

(b) Oblique view

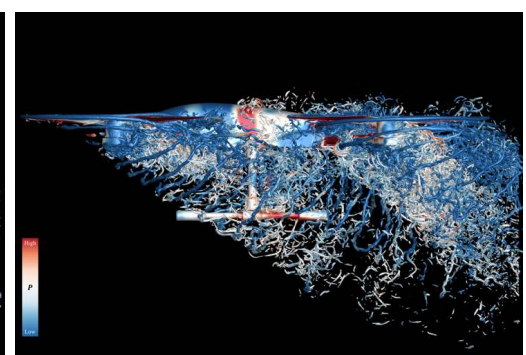

(d) Side view

Figure 17. SUI Endurance quadcopter in forward flight, original design by SUI. The images show the Qcriterion vorticity iso-surfaces colored with the pressure.

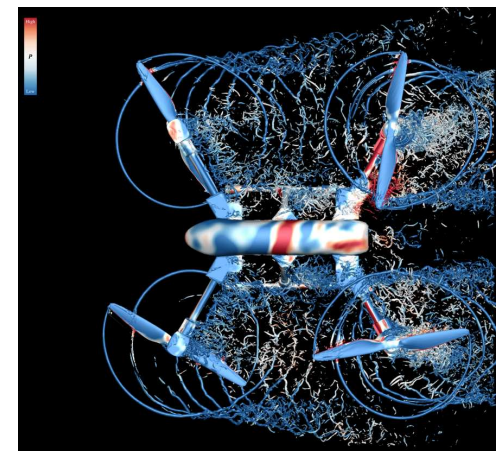

(a) Top view

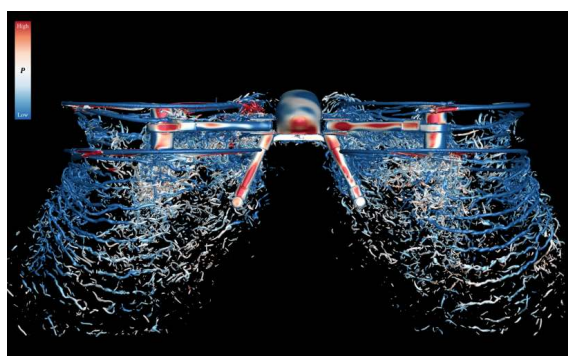

(c) Front view

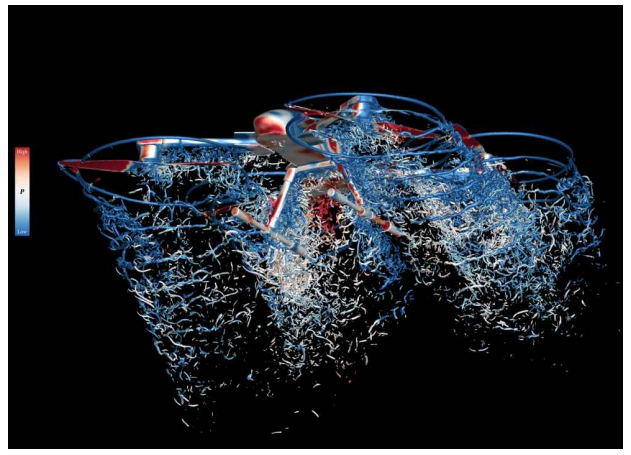

(b) Oblique view

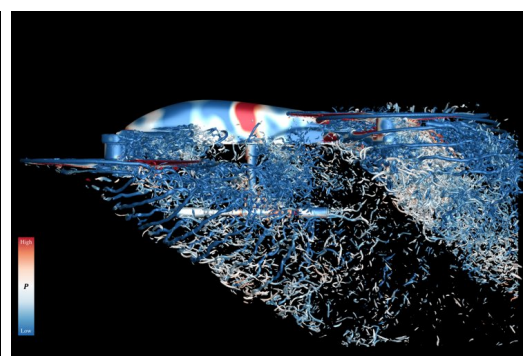

(d) Side view

Figure 18. SUI Endurance quadcopter in forward flight, hybrid configuration. The images show the Q-criterion vorticity iso-surfaces colored with the pressure. 


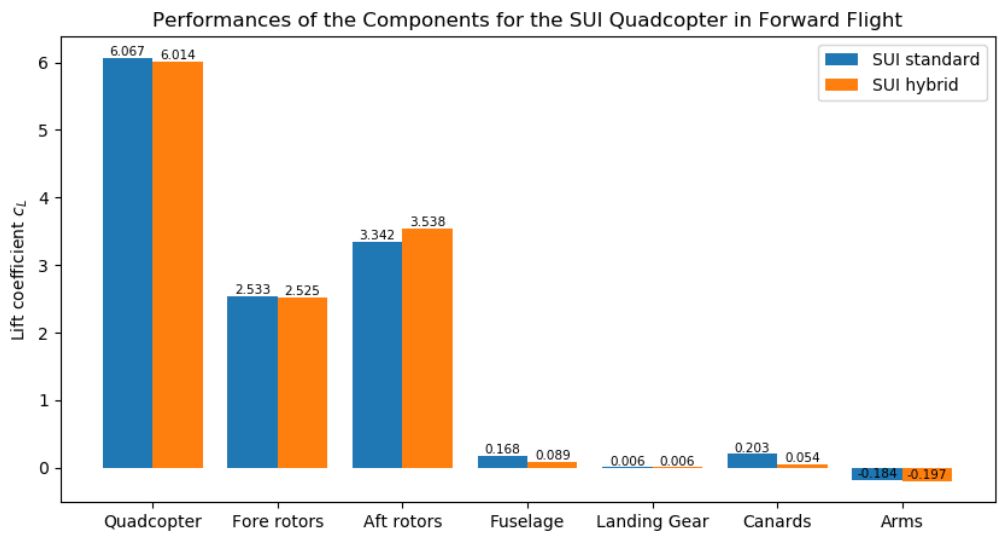

(a) Lift coefficient

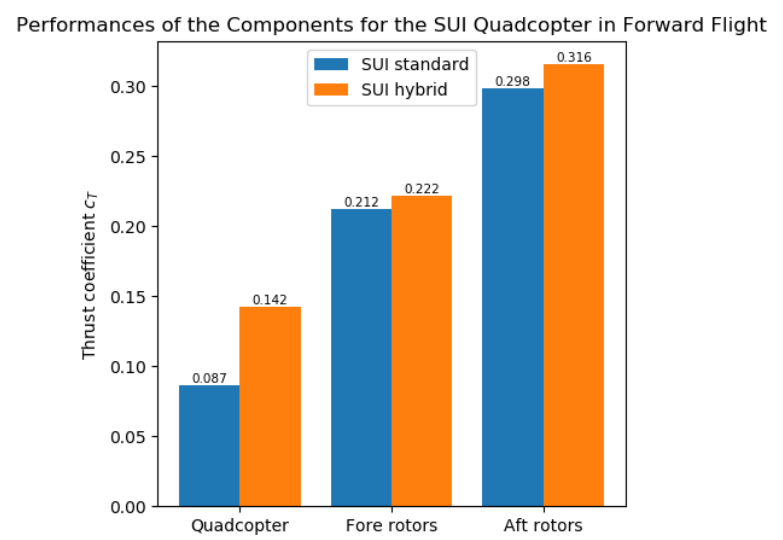

(b) Forward thrust coefficient

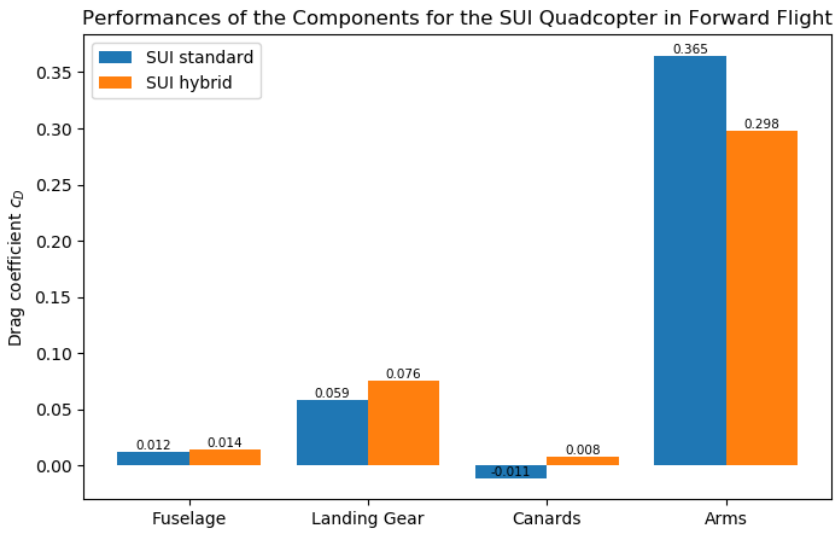

(c) Drag coefficient

Figure 19. Integrated performances of the SUI Endurance quadcopter and its components. The quadcopter performances are the sum of the fore rotors, aft rotors, fuselage, landing gear, canards, and arms. The standard configuration is shown in blue and the hybrid configuration is shown in orange. All coefficients are normalized by the thrust coefficient of a single SUI rotor in hover. 


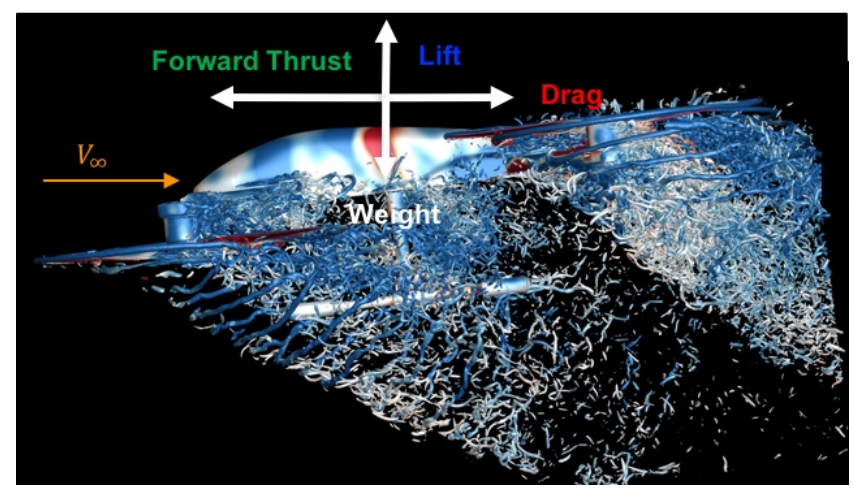

Figure 20. Forces acting on the quadcopter during forward flight.

Figure 21 shows the velocity magnitude in a slice at the rotors for the SUI standard and the SUI hybrid.

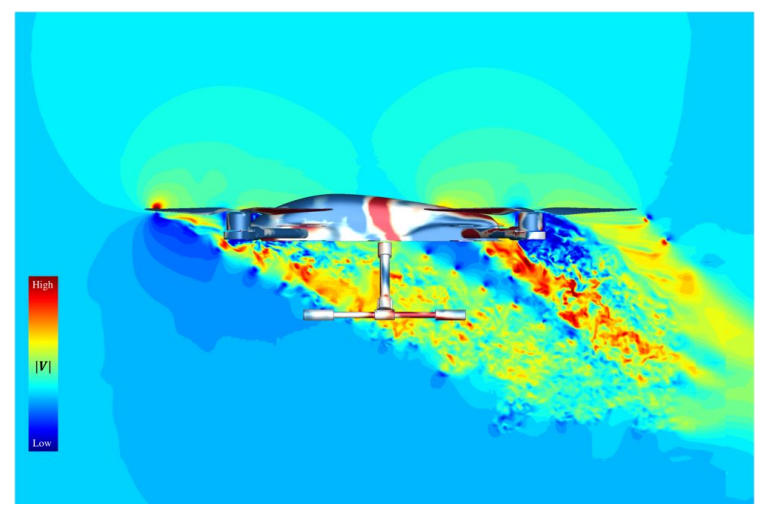

(a) SUI standard

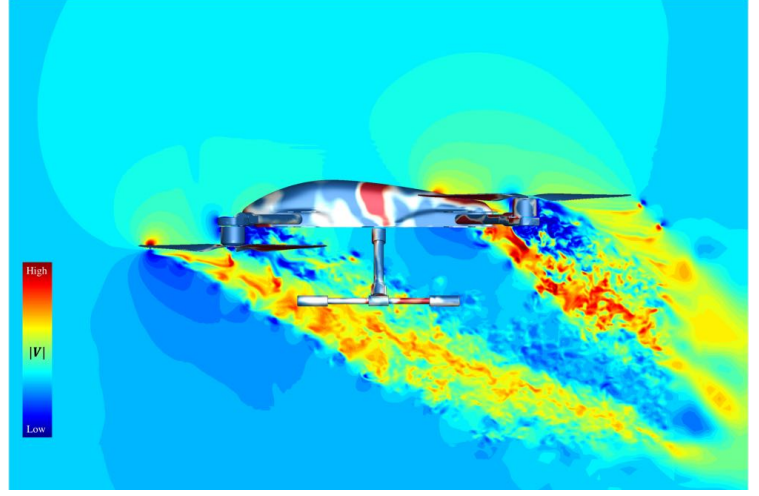

(b) SUI hybrid

Figure 21. Velocity magnitude at a $y$ constant slice for the SUI standard, (a), and for the SUI hybrid, (b). Pressure shown at the body surface.

Previous studies of under-mount rotors ${ }^{12}$ showed a reduction of $1 \%$ in lift and higher levels of noise in hover for under-mount rotors. However, if the quadcopter is going to fly most of its flight envelope in forward flight, the best configuration is the hybrid with under-mount fore rotors and over-mount aft rotors. An increase of more than $60 \%$ in forward thrust directly results in an increase in the range of the vehicle, making it able to cover larger distances for the same battery charge.

\section{Elytron 4S UAV}

The last vehicle of this study is the Elytron $4 \mathrm{~S}$ UAV, the UAV version of the UAM Elytron 4S. The Elytron $4 \mathrm{~S}$ UAV has been simulated in forward flight or "airplane mode", with the tilt-wing at $\theta_{\text {tilt }}=0^{\circ}$, and in hover or "helicopter mode", with an angle $\theta_{\text {tilt }}=90^{\circ}$ for VTOL.

Figure 22 shows the Q-criterion vorticity iso-surfaces colored by the pressure for the Elytron in airplane mode, for an angle of attack of $A o A=0^{\circ}$ in (a), (b), and (c), and for an $A o A=10^{\circ}$ in images (d), (e), and (f). In forward flight, the nose fan does not rotate. The prop-rotors spin at medium velocity, $N_{\text {prop,medium }}=6500 R P M$. The free-stream velocity simulated is $V_{\infty}=20.4 \mathrm{~m} / \mathrm{s}$. The box-wing reduces the induced drag and enhances structural stiffness. The effect of having joined wings with oversized winglets decreases the wingtip vortices and creates a larger effective aspect ratio, reducing the drag. With the tiltwing concept there is no retreating blade problem as in the helicopter rotor blades in forward flight. This allows faster flight as the rotor blade will not suffer from dynamic stall. For a higher angle of attack, the 
wing tip vortices are stronger. Also, the joined wing affects the rotor wakes, as seen in figures 22(c) and (f): the presence of the upper wing deflects the wake downwards. The change of direction of the rotor wake is more important for higher angles of attack.

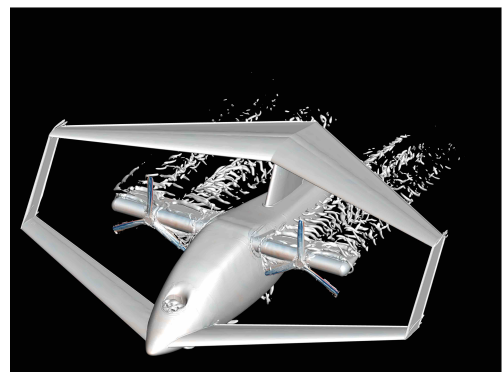

(a) Oblique view, $A \circ A=0^{\circ}$

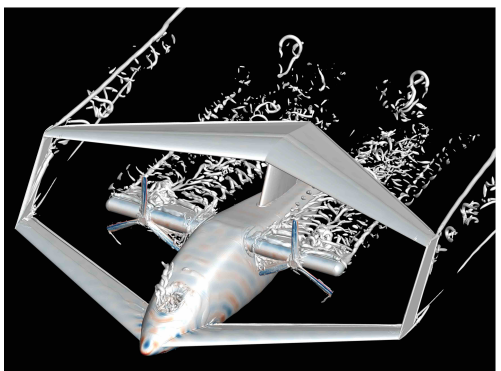

(d) Oblique view, $A o A=10^{\circ}$

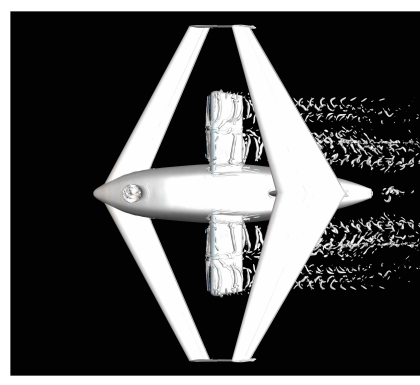

(b) Top view, $A o A=0^{\circ}$

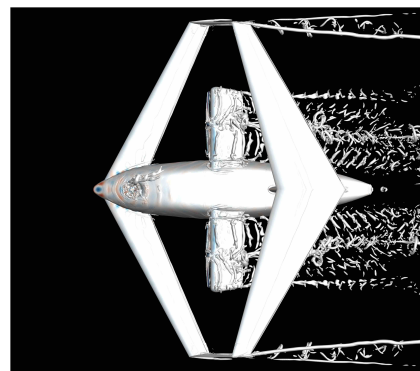

(e) Top view, $A o A=10^{\circ}$

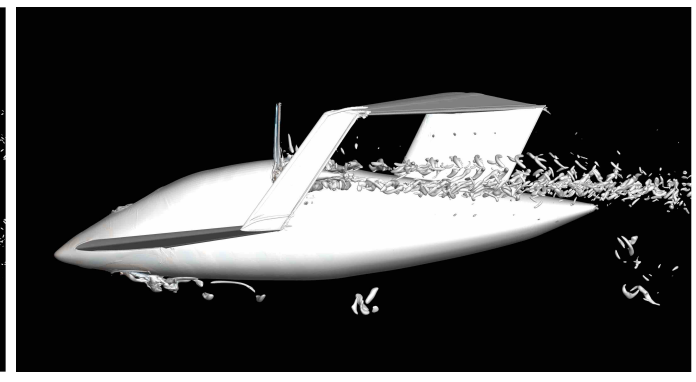

(c) Side view, $A o A=0^{\circ}$

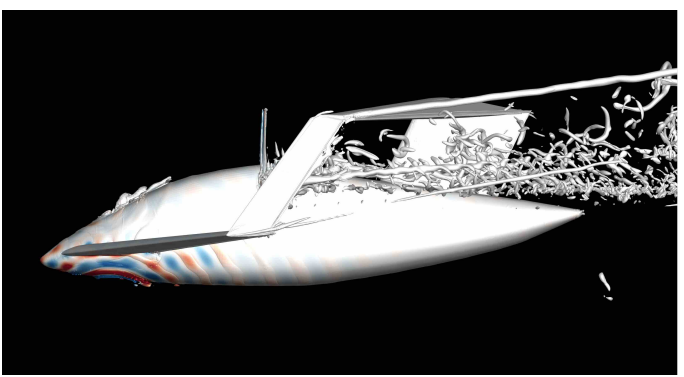

(f) Side view, $A o A=10^{\circ}$

Figure 22. Elytron 4S UAV flow visualization. The images show the Q-criterion vorticity iso-surfaces colored by the pressure in forward flight, for $A o A=0^{\circ}$ (top three images), and $A o A=10^{\circ}$ (bottom three images).

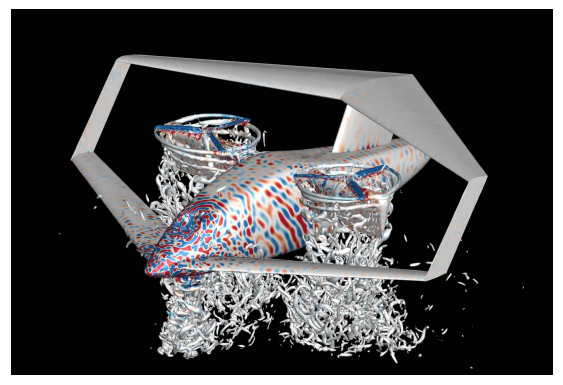

(a) Oblique view.

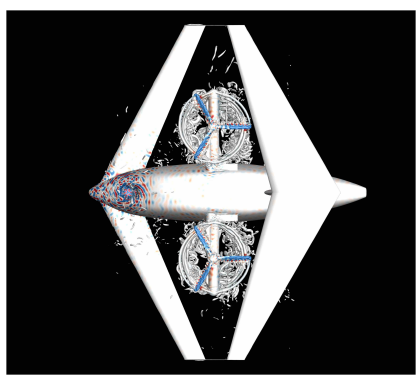

(b) Top view.

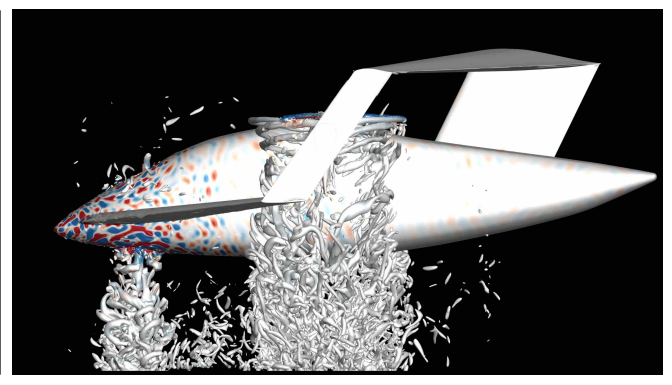

(c) Side view.

Figure 23. Elytron 4S UAV flow visualization. The images show the Q-criterion vorticity iso-surfaces colored by the pressure in hover.

In figure 23 the Elytron is in helicopter mode. In helicopter mode, both the prop-rotors and nose fan rotate at maximum velocity: $N_{\text {fan, } \max }=37000 R P M$ and $N_{\text {prop, } \max }=9000 R P M$. The tilt-wing generates less download force than a tilt-rotor like the XV-15 or the V-22 while in hover. The high rotational velocity of the fan rotor generates strong pressure fluctuations that can be observed along the fuselage. Figure 24 shows the pressure on a plane underneath the vehicle. The pressure waves are very strong, and thus the level of noise is high. The fan and the hole act as a nozzle, accelerating the flow. 


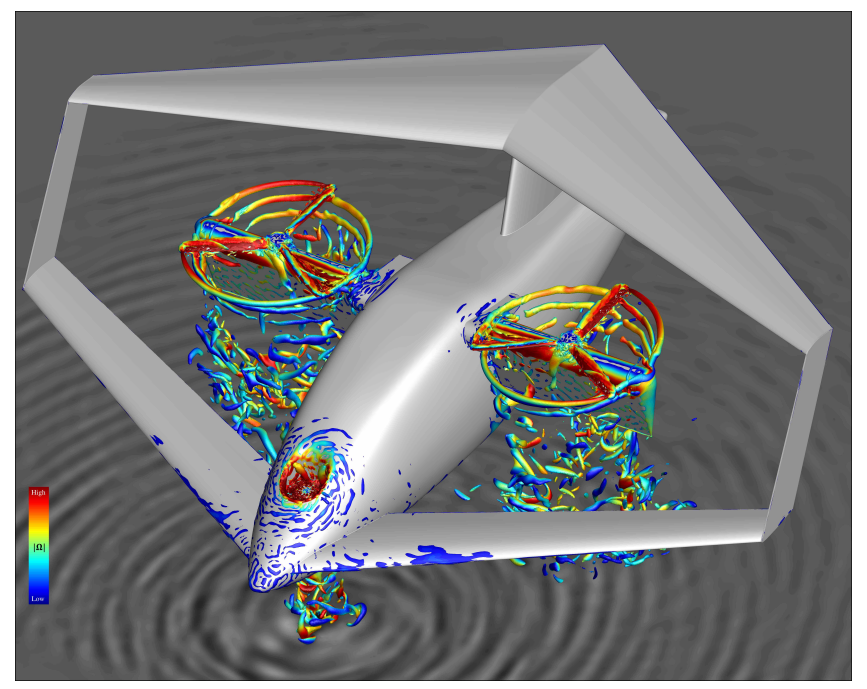

Figure 24. Elytron 4S UAV flow visualization in hover. The images show the Q-criterion vorticity iso-surfaces colored by the vorticity magnitude. The plane below the Elytron shows the pressure waves generated by the high rotational velocity of the fan.

\section{Summary}

High-order accurate Computational Fluid Dynamics simulations have been carried out for several Unmanned Aerial Vehicles. NASA's supercomputers Pleiades and Electra were essential for this work as the overset grids have hundred of millions of grid points. However, only one to two days were needed for converging the quasi-steady solutions using 1024-2048 processors; the solution converged after 30 rotor revolutions.

Adding components underneath the airframe (camera, battery, landing gear) helps by reducing the interactions between rotors, but they must be placed correctly to reduce the fuselage download. In the DJI Phantom 3, the landing gear and battery are immersed in the vortex wake, producing a higher fuselage download than for the simplified airframe and resulting in a reduction of $1 \%$ thrust for the quadcopter. Also, when the rotor-rotor interactions are reduced, the pressure fluctuations are reduced too, resulting in a quieter vehicle. Still, the most important components are the rotors, the sole generators of thrust. The superior design of the original rotor blades generates $26 \%$ more thrust than the replica CF blades.

The effects of weather have also been simulated for the DJI Phantom 3 in hover. Wind gusts unbalance the thrust of individual rotors, producing a moment that, if not compensated, will destabilize the quadcopter, possibly making it crash. We have observed a linear tendency of the moment generated as a function of the wind velocity.

The SUI Endurance, a quadcopter specialized for forward flight, has also been simulated. The fore rotor wakes affect the aft rotors, reducing their performance. The interactions are more important as we increase the forward velocity and angle of attack. A new design from the authors, the hybrid configuration, has been compared with the original configuration, with an impressive increase in forward thrust of $63 \%$.

Finally, the innovative configuration of the Elytron $4 \mathrm{~S}$ UAV has been simulated in forward flight for two different angles of attack and in hover with the nose fan rotating at maximum velocity. 


\section{Acknowledgments}

The authors would like to thank Thomas Pulliam, William Chan, Alan Wray, Yen Liu and Nagi N. Mansour for helpful discussions. High-resolution laser scanning was performed by Eddie Solis of NASA Ames Research Center. This work was supported by DELIVER (Colin Theodore, project manager) and RVLT (Susan Gorton, project manager) projects and utilized the Pleiades and Electra supercomputers at NASA's Advanced Supercomputing (NAS) Division.

\section{References}

${ }^{1}$ Yoon, S., Chan, W. M., and Pulliam, T. H., Computations of Torque-Balanced Coaxial Rotor Flows, AIAA Paper 20170052, The 55th AIAA Aerospace Sciences Meeting, Gaylord, Texas, Jan. 2017.

${ }^{2}$ Yoon, S., Ventura Diaz, P., Boyd, D. D., Chan, W. M., and Theodore, C. R., Computational Aerodynamic Modeling of Small Quadcopter Vehicles, AHS Paper 73-2017-0015, The 73rd Annual AHS International Forum \& Technology Display, Fort Worth, Texas, May 2017.

${ }^{3}$ Nichols, R., Tramel, R., and Buning, P., Solver and Turbulence Model Upgrades to OVERFLOW2 for Unsteady and High-Speed Flow Applications, AIAA Paper 2006-2824, June 2006.

${ }^{4}$ Pulliam, T. H., High Order Accurate Finite-Difference Methods: as seen in OVERFLOW, AIAA Paper 2011-3851, June 2011.

${ }^{5}$ Chan, W. M., Developments in Strategies and Software Tools for Overset Structured Grid Generation and Connectivity, AIAA Paper 2011-3051, Honolulu, Hawaii, June 2011.

${ }^{6}$ Chan, W. M., Gomez, R. J., Rogers, S. E., Buning, P. G., Best Practices in Overset Grid Generation, AIAA Paper 2002-3191, St. Louis, Missouri, June 2002.

${ }^{7}$ Zawodny, N. S., Boyd, D. D., and Burlwy, C. L., Acoustic Characterization and Prediction of Representative, Small-Scale Rotary-Wing Unmanned Aircraft System Components, The $72^{\text {nd }}$ AHS Annual Forum, West Palm Beach, Florida, May 2016.

${ }^{8}$ Russell, C., Jung, J., Willink, G., and Glasner, B., Wind Tunnel and Hover Perfomance Test Results for Multicopter UAS Vehicles, The $72^{\text {nd }}$ AHS Annual Forum, West Palm Beach, Florida, May 2016.

${ }^{9}$ Yoon, S., Pulliam, T. H., and Chaderjian, N. M., Simulations of XV-15 Rotor Flows in Hover Using OVERFLOW, The 5th Decennial AHS Aeromechanics Specialists' Conference, San Francisco, California, Jan. 2014.

${ }^{10}$ Yoon, S., Chaderjian, N. M., Pulliam, T. H., and Holst, T. L., Effect of Turbulence Modeling on Hovering Rotor Flows, AIAA Paper 2015-2766, The 45th AIAA Fluid Dynamics Conference, Dallas, Texas, June 2015.

${ }^{11}$ Yoon, S., Lee, H. C., and Pulliam, T. H., Computational Analysis of Multi-Rotor Flows, AIAA Paper 2016-0812, The 54th AIAA Aerospace Sciences Meeting, San Diego, California, Jan. 2016.

${ }^{12}$ Yoon, S. Lee, H. C., and Pulliam, T. H., Computational Study of Flow Interactions in Coaxial Rotors, The AHS Technical Meeting on Aeromechanics Design for Vertical Lift, San Francisco, California, Jan. 2016.

${ }^{13}$ Jespersen, D., Pulliam, T. H., and Buning, P., Recent Enhancements to OVERFLOW, AIAA Paper 97-0644, Reno, Nevada, Jan. 1997.

${ }^{14}$ Lakshminarayan, V. K., and Baeder, J. D., Computational Investigation of Micro Hovering Rotor Aerodynamics, Journal of the American Helicopter Society, 55, 022001, 2010.

${ }^{15}$ Spalart, P. R., Jou, W-H., Strelets, M., and Allmaras, S. R., Comments on the Feasibility of LES for Wings and on a Hybrid RANS/LES Approach, Advances in DNS/LES, Greyden Press, 1997, pp. 137-147.

${ }^{16}$ Leishman, J. G., Principles of helicopter aerodynamics (2nd edition), Cambridge University Press, 2006.

${ }^{17}$ Smagorinsky, J., General circulation experiments with the primitive equations, I. The basic experiment. Monthly Weather Review, 91: pp 99-164, 1963.

${ }^{18}$ Spalart, P. R., Strategies for Turbulence Modeling and Simulations, International Journal of Heat and Fluid Flow, 21, 2000, pp. 252-263.

${ }^{19}$ Otsuka, H., and Nagatani, K., Reduction of Pitching Moment Generation of a Quadrotor UAV in Gust with Slant Rotors, AIAA Paper 2017-0500, AIAA SciTech Forum and the 55th AIAA Aerospace Sciences Meeting, January 2017, Grapevine, Texas. 